\title{
RESPONSE OF STEVIA (STEVIA REBAUDIANA L.) TO BIOGAS FERTILIZER AND NATURAL EXTRACTS UNDER SOUTH SINAI CONDITIONS
}

\author{
Eman M. Abou El-Ghait", Y.F.Y. Mohamed*, W.M. Abd El-Azim** and M. R. Mansour** \\ ${ }^{*}$ Horticulture Dept., Fac. Agric., Benha University, Egypt \\ ${ }^{* *}$ Medicinal and Aromatic Plants Department, Desert Research Center, Cairo, Egypt
}

\begin{abstract}
Two field experiments were conducted in 2019 and 2020 seasons at Desert Research Center, Mataria, Cairo, Egypt, and its experimental farm at Ras Sudr Experimental Station in South Sinai, in cooperation with the Department of Horticulture, Faculty of Agriculture Benha University, to study the effect of biogas slurry fertilizer and natural extracts on biomass, chemical constituents, and quality of Stevia rebaudiana L. plant. Results showed that biogas fertilizer along with the different natural extracts exerted a significant
\end{abstract}

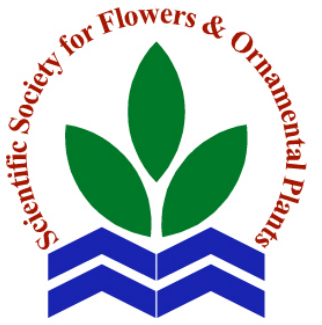

Scientific J. Flowers \& Ornamental Plants, 8(3):375-400 (2021).

Received:

$17 / 8 / 2021$

Accepted:

$1 / 9 / 2021$

Corresponding author: M.R. Mansour mohmedrashed87@gmail.com

\section{INTRODUCTION}

Stevia (Stevia rebaudiana L.) is a green herb plant that belongs to family Asteraceae its leaves contain several chemical substances called glycosides in addition, it contains volatile oils (Hossain et al., 2017). These glycosides have a sweet taste but do not contain any calories. The main glycoside is known as stevioside, it is 300-400 times sweeter than sucrose (Singh et al., 2019; Talevi, 2021). Chemical fertilizers have been indispensable in agriculture, but they are currently prohibitively costly and, in some cases, unavailable on time (Naguib, 2011). The combined use of organic and chemical 
fertilizers can minimize dependency on costly chemical inputs. To maintain high crop yields while maintaining soil fertility, it is crucial to produce an optimal fertilizer and manure combination in the crop (Roba, 2018).

Biogas slurry (BGS) is a byproduct of biogas processing using cow dung. Biogas slurry is an excellent source of plant nutrients and can enhance soil properties (Garg et al., 2005). According to (Khan et al., 2015), biogas slurry contains many rich and nutritive elements such as N, P, K, and trace elements ( $\mathrm{Zn}, \mathrm{Fe}, \mathrm{Cu}, \mathrm{Ni}$, and so on). Farmers must use chemical fertilizer to increase crop yield, however, if only mineral fertilizers are added to the soil without the addition of organic manure, land productivity would decrease, (Sonklien et al., 2020)

Farmers and researchers are currently focusing heavily on bio stimulants to increase agricultural sustainability; nevertheless, additional natural products such as (yucca, Azolla, and garlic extracts), should be examined, studied, and appraised to improve agricultural sustainability, namely crop quality and quantity. Several prior research found that plant extracts affected hormones, organic acids (Abou Chehade et al., 2018), and polyphenols (Lucini et al., 2018).

The garlic extract is made from the sap of the garlic bulb Allium sativum (L.), which belongs to the Liliaceae family. It is characterized by a high concentration of Sulphur-containing amino acids, such as cysteine and methionine (Synge, 1970). Garlic also includes volatile oil, allicin, alliin, sugar, iodine, and vitamins are among the products used (Al Mayahi and Fayadh, 2015). According to Abou Hussein et al. (1975 a) and Abou Hussein et al. (1975 b). Garlic extract has a wide range of effects due to its hormonal origin, which plays an important role in cell lateral extension and elongation. Concerning the garlic acid effect, treatment of Majorana hortensis and Salvia officinalis with garlic extract at 50 or 100 percent concentration increased fresh and dry weights, photosynthetic pigments of chlorophylls $\mathrm{a}$ and $\mathrm{b}$, and total soluble carbohydrates content in the first and second cut, as well as total oil content (El-Rokiek et al., 2019)

Azolla has a high nitrogen content and has been used as an environmentally safe fertilizer for wetland rice production, as well as for its ability to retain significant quantities of nutrients (Enwall et al., 2005). It is one of the additional types of fertilizer used in addition to other bio-fertilizers. (Rabie et al., 2020) on Matricaria chamomilla concluded that the addition of compost and $50 \%$ of chemical fertilizer to Azolla extract at $50 \%$ as foliar (spray) have a positive effect on chemical constituents except for nitrate and nitrite concentrations.

Yucca schidigera, a plant that grows in some of the harshest conditions. It is a natural extract of yucca which is abundant in steroidal saponins, as well as other stressrelieving compounds and antioxidants. When added to the soil with liquid starters, Yucca extract acts as a wetting agent, minimizing the formation of dry spots (Piacente et al., 2005). So, the aim of this study is to improve the yield, chemical constituents and quality of Stevia rebaudiana L. plant by using biogas slurry as organic fertilizer and natural extracts under the conditions of sandy soil in south Sinai.

\section{MATERIALS AND METHODS}

Two field experiments were conducted in 2019 and 2020 seasons at Desert Research Center, Mataria, Cairo Egypt, and its Experimental Farm at Ras Sudr Experimental Station in South Sinai, to study the effect of biogas fertilizer and natural extracts on the biomass, chemical composition, and quality of the Stevia rebaudiana $\mathrm{L}$. plant. The experimental site is located in arid conditions; The annual rainfall of $15 \mathrm{~mm}$ rainfall ranges to $30 \mathrm{~mm}$ and occurs during a short period from November to March. The average annual relative humidity is $57.2 \%$, and it represents 
the highest temperature during the year that may reach $43{ }^{\circ} \mathrm{C}$, the average maximum temperature during the year is $27.5^{\circ} \mathrm{C}$ and the average minimum temperature is $15.2{ }^{\circ} \mathrm{C}$ during the two successive seasons of 2018/19 and 2020. The physical and chemical properties of the soil sample were determined, according to (Rathje, 1959), and are shown in Table $(1,2)$. Drip irrigation lines were installed within $20 \mathrm{~cm}$ between drippers with an average of $4 \mathrm{~L} / \mathrm{h}$. Underground water was used as an irrigation source analyses of the irrigation water samples were determined according to (Rayment and Lyons, 2010) and their properties are shown in Table (3).

Seeds of Stevia were gained from Stevia Farm Egypt Co., Cairo, Egypt, which were harvested in early November of the previous season. The seeds were sown on January $1^{\text {st }}$ in a mixture of $3: 1 \mathrm{v} / \mathrm{v}$ peat moss: vermiculite. The seed trays were placed in polyethylene greenhouses and irrigated thoroughly until germination, then the seedlings trays were transferred to saran (63\% shade) until reached acceptable size (7 $\mathrm{cm}$ height with 2-3 pairs of true leaves). The seedlings were cultivated in the field on $15^{\text {th }}$ April. The experimental plot was $5.85 \mathrm{~m}^{2}$ $(20 \times 70 \mathrm{~cm})$, all plots included eight rows each row was $70 \mathrm{~cm}$ apart and twenty meters in length. The treatments were arranged in a split-plot design with three replicates.

The main plots were occupied by biogas and chemical fertilizers levels treatment $(\mathrm{T})$ in randomly distributed, while the sub-plot was occupied by natural extracts of Azolla, garlic and yucca as foliar and drench application treatments $(\mathrm{M})$ in randomly arranged Main plots $\left(\mathrm{T}_{1}, \mathrm{~T}_{2}, \mathrm{~T}_{3}, \mathrm{~T}_{4}\right)$ :

\section{Main Plot:}

$\mathrm{T}_{1}: 100 \%$ recommended dose (RD) of NPK $(100: 50: 50 \mathrm{Kg} / \mathrm{ha})$.

$\mathrm{T}_{2}: 100 \%$ recommended dose of biogas slurry (BGS) $(6000 \mathrm{~kg} / \mathrm{ha})$.

$\mathrm{T}_{3}: 50 \%$ recommended dose $(\mathrm{RD})$ of $\mathrm{NPK}+$ biogas slurry (BGS) $(5000 \mathrm{~kg} / \mathrm{ha})$.

$\mathrm{T}_{4}: 50 \%$ recommended dose $(\mathrm{RD})$ of NPK + biogas slurry (BGS) (3000 kg /ha).

Table 1. The chemical properties of the experimental soil.

\begin{tabular}{|c|c|c|c|c|c|c|c|c|c|c|}
\hline \multirow{2}{*}{ pH } & \multirow{2}{*}{$\begin{array}{c}\text { E.C. } \\
\text { (ds/cm) }\end{array}$} & \multirow{2}{*}{$\begin{array}{c}\text { O.M. } \\
(\%)\end{array}$} & \multicolumn{4}{|c|}{ Cations (meq/l) } & \multicolumn{4}{|c|}{ Anions (meq/l) } \\
\hline & & & $\mathbf{C a}^{++}$ & $\mathbf{M g}^{++}$ & $\mathrm{Na}^{+}$ & $\mathbf{K}^{+}$ & $\mathrm{HCO}_{3}^{-}$ & $\mathrm{SO}_{4}^{--}$ & $\mathrm{Cl}^{-}$ & $\mathrm{CO}_{3}^{-}$ \\
\hline \multirow{4}{*}{7.95} & \multirow{4}{*}{3.14} & \multirow{4}{*}{0.7} & 3.44 & 8.95 & 3.43 & 1.8 & 3.22 & 3.48 & 10.92 & 0 \\
\hline & & & \multicolumn{4}{|c|}{ Available macronutrients $(\mathrm{mg} / \mathrm{kg})$} & \multicolumn{4}{|c|}{ Available micronutrients (mg/kg) } \\
\hline & & & $\mathbf{N}$ & $\mathbf{P}$ & $\mathbf{K}$ & $\mathrm{Ca}$ & $\mathbf{F e}$ & Mn & $\mathbf{C u}$ & $\mathbf{Z n}$ \\
\hline & & & 60 & 27.24 & 73 & 306 & 94.92 & 12.92 & 17.4 & 3.88 \\
\hline
\end{tabular}

Table 2. Physical properties of the experimental soil.

$\begin{array}{ccccc}\text { Coarse Sand (\%) } & \text { Fine Sand (\%) } & \text { Silt (\%) } & \text { Clay (\%) } & \text { Soil texture } \\ 46 & 50 & 3 & 1 & \text { sandy }\end{array}$

Table 3. Water analysis of the irrigation water.

\begin{tabular}{cccccccccc}
\hline \multirow{2}{*}{$\mathbf{p H}$} & $\begin{array}{c}\text { E.C. } \\
(\mathbf{d s} / \mathbf{c m})\end{array}$ & $\mathbf{C a}^{++}$ & \multicolumn{4}{c}{ Cations (meq/l) } & \multicolumn{4}{c}{ Anions (meq/l) } \\
& $\mathbf{M g}$ & $\mathbf{N a}^{+}$ & $\mathbf{K}^{+}$ & $\mathbf{C O}^{--}$ & $\mathbf{H C O}_{3}^{-}$ & $\mathbf{S O}_{4}^{--}$ & $\mathbf{C l}^{-}$ \\
\hline 7.95 & 0.8 & 3.09 & 0.8 & 0.93 & 0.48 & 0 & 2.16 & 1.57 & 1.90 \\
\hline
\end{tabular}


Sub Plot:

$\mathrm{M}_{1}$ : Azolla aqueous extract (50\%) as a foliar spray.

$\mathrm{M}_{2}$ : Azolla aqueous extract (50\%) as drench addition.

M3: Garlic aqueous extract (10\%) as a foliar spray.

M4: Garlic aqueous extract (10\%) as drench addition.

M5: Yucca aqueous extract $(0.3 \mathrm{~g} / \mathrm{l})$ as a foliar spray.

M6: Yucca aqueous extract $(0.3 \mathrm{~g} / \mathrm{l})$ as drench addition.

$\mathrm{M}_{7}$ : Distilled water as a foliar spray.

M8: Distilled water as drench addition.

The experiment was conducted for six months from April to October for each season.

\section{Biogas slurry fertilizer (BGS):}

Biogas doses were added during the site and row preparation in assigned experimental units nitrogen, phosphates, and potassium fertilizers as ammonium nitrate $(33 \% \mathrm{~N})$ at the rate of $100 \mathrm{~kg} /$ hectare, Calcium superphosphate $\left(15.5 \% \mathrm{P}_{2} \mathrm{O}_{5}\right)$ at the rate of $50 \mathrm{~kg} /$ hectare and potassium sulphate $\left(48 \% \mathrm{~K}_{2} \mathrm{O}\right)$ at the rate of $50 \mathrm{~kg} /$ hectare, respectively were added to assigned experimental units. Half of the nitrogen dose and the entire doses of phosphorus and potassium were added as a basal dose during assigned experimental rows preparation and the remaining nitrogen was applied after second cut, other treatments of natural aqueous extracts were applied as a foliar spray as well as drench (dressing application) in the early morning starting 15 days after transplanting seedlings at onemonth Intervals using biofilm $1 \mathrm{~g} \mathrm{l}^{-1}$ as a wetting agent and hand pump sprayer and nozzle in both Azolla and Garlic aqueous extracts .

The biogas slurry fertilizer (BGS) used in the experiment was obtained from the Land and Water Research Institute, Agriculture Research Center. The chemical characteristics of biogas slurry are given in Table (4).

\section{Natural Extracts preparation:}

\section{Garlic extract:}

The garlic extract (Allium sativum) was prepared according to (Elzaawely et al., 2018) as $100 \mathrm{~g}$ of freshly grown cloves of garlic were brought and were macerated in $100 \mathrm{ml}$ of tap water in a tightly stoppered beaker and kept in a freezer for 24 hours at $\left(20^{\circ} \mathrm{C}\right)$. The macerate was then thawed by allowed to melt at room temperature $\left(25^{\circ} \mathrm{C}\right)$. The melted mixture was blended in a blender for 5 minutes, then the blended macerate was frozen and melted twice. The aqueous macerate extract was kept in the refrigerator $\left(1{ }^{\circ} \mathrm{C}\right)$ as crude extract for 10 hours and then the blended mixture was filtered through a double layer of cheesecloth. The obtained filtrates were completed by distilled water to a final volume of 1 liter. The chemical constituents of aqueous extract were shown in Table (5).

\section{Azolla extract:}

Azolla was obtained from the Land and Water Research Institute, Agriculture Research Center (Table, 6). Azolla extract was extracted from fresh plant parts of Azolla depending on the extraction method according to (Wilson and Al-Hamdani, 1997) with slight modification. Firstly, washing the fresh plant part with tap water, followed by distilled water, then put in plastic bags with sterilized distilled water at a rate of 1:1 (weight/volume) and kept in the freezer for at least 12 hours at $-20{ }^{\circ} \mathrm{C}$.

\section{Table 4. chemical constituents of biogas} fertilizer.

\begin{tabular}{lcc}
\hline \multirow{2}{*}{ Biogas manure } & \multicolumn{2}{c}{ Values } \\
& First season & Second season \\
\hline Organic matter \% & 34 & 33 \\
Organic carbon \% & 20 & 23 \\
Total nitrogen \% & 1.65 & 1.64 \\
Total phosphate \% & 0.85 & 0.9 \\
Total potassium \% & 0.35 & 0.34 \\
Density Kg/m & 285 & 260 \\
Moisture \% & 10 & 10 \\
Saturation \% & 210 & 210 \\
\hline
\end{tabular}


Table 5. Chemical constituents of garlic aqueous extracts fresh cloves.

\begin{tabular}{llc}
\hline Constituents & & Quantitative Analysis \\
\hline Potassium & & $2,127 \mathrm{ppm}$ \\
Calcium & & $35.36 \mathrm{ppm}$ \\
Magnesium & & $104.65 \mathrm{ppm}$ \\
Zinc & & $2.84 \mathrm{ppm}$ \\
Phosphorous & & $600.3 \mathrm{ppm}$ \\
Aluminum & & $2.315 \mathrm{ppm}$ \\
& IAA & $210.80 \mathrm{ng} \mathrm{ml}^{-1} \mathrm{fw}$ \\
Auxins & IBA & $304.15 \mathrm{ng} \mathrm{ml}^{-1} \mathrm{fw}$ \\
& IPA & $627.21 \mathrm{ng} \mathrm{ml}^{-1} \mathrm{fw}$ \\
Cytokinins & tZ & $54.09 \mathrm{ng} \mathrm{m}^{-1} \mathrm{fw}$ \\
& tZR & $76.47 \mathrm{ng} \mathrm{m}^{-1} \mathrm{fw}$ \\
& GA & $113.72 \mathrm{ng} \mathrm{ml}^{-1} \mathrm{fw}$ \\
Gibberellins & GA$_{4}$ & $68.53 \mathrm{ng} \mathrm{ml}^{-1} \mathrm{fw}$ \\
& GA $_{7}$ & $173.52 \mathrm{ng} \mathrm{ml}^{-1} \mathrm{fw}$ \\
\hline
\end{tabular}

Table 6. Chemical composition of fresh Azolla extracts.

\begin{tabular}{lc}
\hline Properties & $\begin{array}{c}\text { Quantitative } \\
\text { analysis }\end{array}$ \\
\hline PH & 6.35 \\
EC & 1.01 \\
Total nitrogen (ppm) & 150 \\
Total Phosphorus (P2O5; ppm) & 34 \\
Total potassium $\left(\mathbf{K}_{\mathbf{2}} \mathbf{O}\right)(\mathbf{p p m})$ & 152 \\
Chemical oxygen demand $(\mathbf{C O D} ; \mathbf{m g} / \mathbf{l})$ & 12 \\
Biological oxygen demand $(\mathrm{BOD} ; \mathbf{m g} / \mathbf{l})$ & 3.1 \\
\hline
\end{tabular}

The plant material was then withdrawn from the refrigerator and allowed to melt at room temperature. The mixture was hardly crushed and blended for 5 minutes, filtered through double layers of cheesecloth, centrifuged at 12,000 rpm for 30 minutes. The resulting solution was kept in the refrigerator at $5{ }^{\circ} \mathrm{C}$ until use. The crude extract was considered $100 \%$ concentration and serial dilution $(50 \%)$ was performed using distilled water. (El-Shimi et al., 2015).

\section{Yucca extract:}

Yucca extract powder was obtained from NPK industries company it was prepared by dissolving $0.3 \mathrm{~g}$ of the powder in 11 water as the chemical composition of yucca extract id shown in Table (7).

\section{Harvesting:}

The experiments were conducted for six months from April to October of each season. The first cut was conducted on the July $15^{\text {th }}$ and the second cut was conducted on October $15^{\text {th }}$ of both seasons.
Table 7. Chemical composition of yucca extracts.

\begin{tabular}{lc}
\hline \multicolumn{1}{c}{ Constituents } & $\begin{array}{c}\text { Quantitative analysis } \\
\mathbf{m g} \mathbf{1 0 0} \mathbf{~ g}^{-1} \mathbf{~ d w}\end{array}$ \\
\hline Potassium & 279 \\
Phosphorous & 27.8 \\
Magnesium & 21.65 \\
Manganese & 0.40 \\
Zinc & 0.35 \\
Coper & 0.10 \\
Vitamin c & 21.2 \\
Vitamin B6 & 0.10 \\
Vitamin K & 1.95 \\
Thiamine & 0.10 \\
Riboflavin & 0.50 \\
Niacin & 0.90 \\
\hline
\end{tabular}

\section{Biomass characteristics measure:}

Shoots fresh weight biomass $\mathrm{kg} / \mathrm{ha}$, shoots dry weight biomass $\mathrm{kg} / \mathrm{ha}$ and leaves fresh weight biomass $\mathrm{kg} / \mathrm{ha}$ Leaves dry weight biomass $\mathrm{kg} / \mathrm{ha}$.

\section{Chemical constituent attributes:}

\section{Photosynthetic pigments determination:}

Chlorophyll a, b, total chlorophyll, and carotenoids were determined following (Saric, 1967).

\section{Nutrient element\%:}

Nitrogen was determined by the modified micro-Kjeldahl method as described by (A.O.A.C., 1980). Phosphorus was determined using the ammonium molybdate method according to (Murphy and Riley, 1962). Potassium \% was estimated using flam photometric method according to (Cottenie et al., 1982).

\section{Nitrate concentration:}

Stevia sampled from the leaves was dried by air to determine nitrate concentration. Nitrate levels in the dry leaves were determined according to the method of (Cataldo et al., 1975).

\section{Stevioside content:}

The sativoside content in the leaves was estimated by HPLC analysis according to. (Abou-arab et al., 2010). 


\section{Experimental design:}

The experimental layout was a split-plot design arranged with three replicates. Each replicate contained 32 treatments, chemical and three biogas fertilizer levels treatment $(\mathrm{T})$ were assigned in main plots, which were split into eight treatments of natural extracts and five plants were used as an experimental unit. Collected data of both seasons were pooled and the obtained results were analyzed using MSTATC Program.(Bricker, 1991) Means were compared using LSD test at 0.05 level according to (Snedecor and Cochran, 1989).

\section{RESULTS}

\section{Shoots fresh and dry weights biomass:}

According to data presented in Table (8), all fertilization treatments succeeded in increasing the shoots fresh and dry weights Biomass of Stevia rebaudiana, particularly the recommended dose of chemical fertilizer $\left(\mathrm{T}_{1}\right)$ in the first cut of both seasons. Although the second cut follows the same pattern as the first cut of both seasons, followed by $50 \% \mathrm{NPK}+5000 \mathrm{~kg} \mathrm{~h}^{-1}\left(\mathrm{~T}_{3}\right)$ with no major differences. On the other hand, plants treated with the (T2) prescribed dose of biogas fertilizer of both cuts and seasons, produced the lowest fresh and dry weights Biomass.

The results achieved concerning an increment in shoots fresh and dry weights biomass by different natural extracts application methods, garlic aqueous extract as drench addition at $10 \%\left(\mathrm{M}_{4}\right)$ recorded the highest shoots fresh weight biomass in the first cut of both seasons followed by Azolla aqueous extract at (50\%) as drench addition with no significant differences between them in the second cut. Although the shoots dry weights biomass goes in the line with those found in fresh weight biomass as $\left(\mathrm{M}_{4}\right)$ have the highest dry weight biomass values of both $1^{\text {st }}$ and $2^{\text {nd }}$ cuts but without significant variance with $\left(\mathrm{M}_{2}\right)$ of both seasons. At the same time, the lowest values were recorded by distilled water as drenching or foliar application method ( $\mathrm{T}_{7}$ and $\mathrm{T}_{8}$ ) with no significant differences between them.

It is also obvious from Tables that the positive impact of the interaction between the prescribed dose of chemical fertilizer and yucca as a foliar spray $\left(\mathrm{T}_{1} \times \mathrm{M}_{5}\right)$ gave the highest plant fresh and dry weights biomass only in the first cut of both seasons. In the second cut registered the highest shoot fresh and dry weights biomass with 50\% NPK + $5000 \mathrm{~kg} \mathrm{~h}^{-1}$ biogas, combined with the addition of the drenched garlic aqueous extract $\left(\mathrm{T}_{3} \times \mathrm{M}_{4}\right)$ of both seasons. In the contrast, the lightest fresh and dry weights biomass was recorded by the interaction of recommended dose of biogas fertilizer and distilled water as a foliar spray $\left(\mathrm{T}_{2} \times \mathrm{M}_{7}\right)$ in the first cut of both seasons while the second cut was in the same line with the first but with distilled water as drench addition $\left(\mathrm{T}_{2} \times \mathrm{M}_{8}\right)$.

\section{Leaves fresh and dry weights biomass:}

Data presented in Table (9) states that using recommended dose of chemical fertilizer $\left(T_{1}\right)$ recorded the heaviest leaves fresh and dry wights biomass only in the first cut of both seasons. Although the second cut recorded the highest leaves fresh weights of both seasons by $50 \%$ NPK $+5000 \mathrm{~kg} \mathrm{~h}-1$ biogas fertilizer $\left(\mathrm{T}_{3}\right)$. However, in the case of the leaves dry weight biomass there were non-significant differences with $\left(\mathrm{T}_{1}\right)$ in the first season only. Whereas the lightest leaves fresh and dry weights biomass was observed during both seasons by recommended dose of biogas fertilizer $\left(\mathrm{T}_{2}\right)$.

It is quite evident as shown from tabulated data that the response of leaves fresh and dry weights biomass to natural extracts were enhanced by Azolla aqueous extract as drench addition $\left(\mathrm{M}_{2}\right)$ which registered the highest values of both cuts and seasons. However, distilled water as foliar or drench addition $\left(\mathrm{M}_{7}\right.$ or $\left.\mathrm{M}_{8}\right)$ gave the lowest values in this regard.

In both seasons, the response of stevia leaves fresh and dry weights biomass to the interaction of fertilization and natural extract 
Table 8. Effect of biogas, chemical fertilizer, and natural extracts and their interaction on shoots fresh and dry wights biomass of Stevia rebaudiana L. plants in 2019 and 2020 seasons for two cuts.

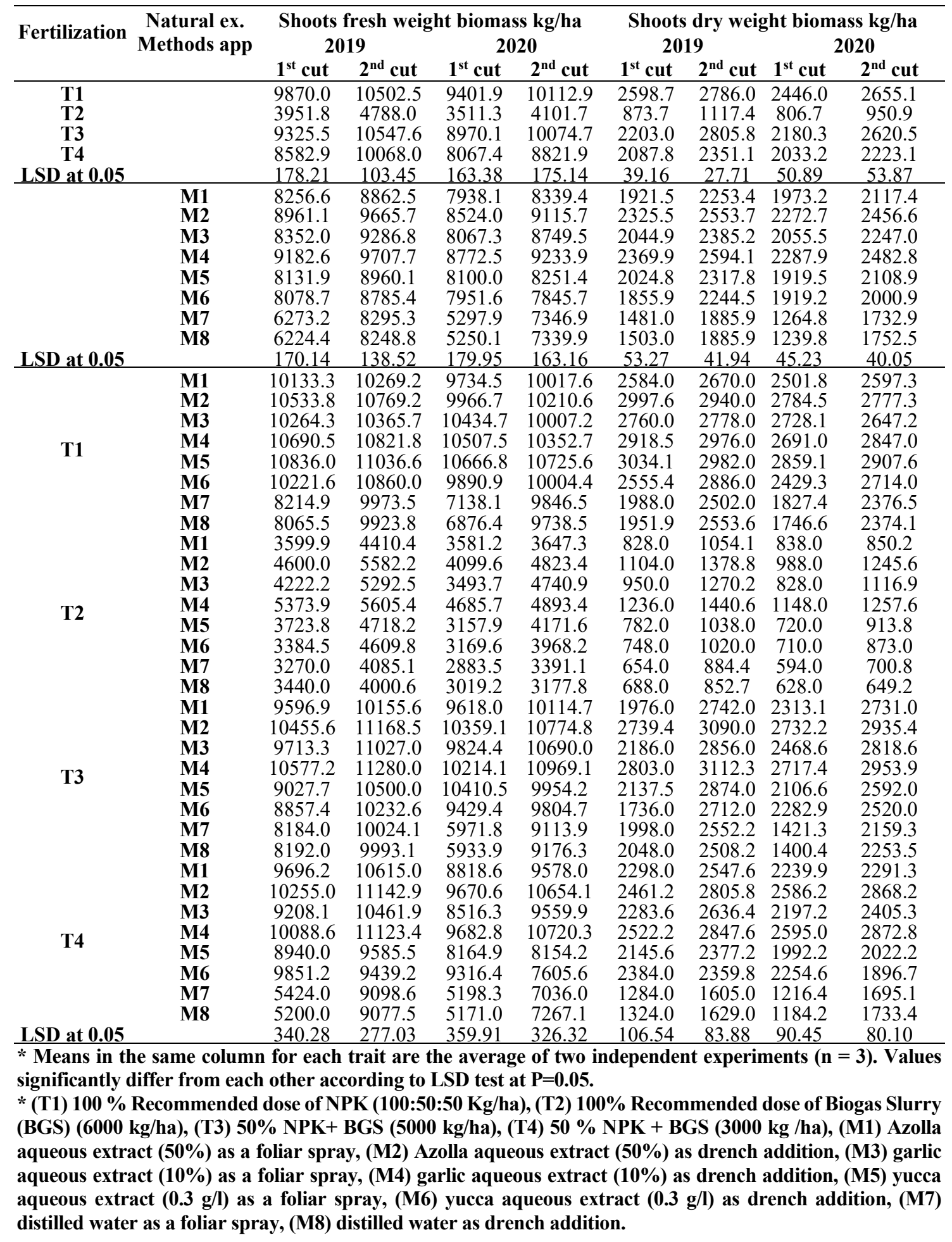


Table 9. Effect of biogas, chemical fertilizer, and natural extracts and their interaction on leaves fresh and dry weights biomass of Stevia rebaudiana L. plants in 2019 and 2020 seasons for two cuts.

\begin{tabular}{|c|c|c|c|c|c|c|c|c|c|}
\hline \multirow{3}{*}{ Fertilization } & \multirow{3}{*}{$\begin{array}{l}\text { Natural ex. } \\
\text { Methods app }\end{array}$} & \multicolumn{4}{|c|}{ Leaves fresh weights biomass $\mathrm{kg} / \mathrm{ha}$} & \multicolumn{4}{|c|}{ Leaves dry weights biomass $\mathrm{kg} / \mathrm{ha}$} \\
\hline & & \multicolumn{2}{|c|}{2019} & \multicolumn{2}{|c|}{2020} & \multicolumn{2}{|c|}{2019} & \multicolumn{2}{|c|}{2020} \\
\hline & & $1^{\text {st }}$ cut & $2^{\text {nd }}$ cut & $1^{\text {st }}$ cut & $2^{\text {nd }}$ cut & $1^{\text {st }}$ cut & $2^{\text {nd }}$ cut & $1^{\text {st }}$ cut & $2^{\text {nd }}$ cut \\
\hline T1 & & 6362.8 & 6450.6 & 6064.4 & 6223.4 & 1722.3 & 1745.2 & 1641.5 & 1682.8 \\
\hline T2 & & 1982.1 & 2613.6 & 1790.9 & 2114.7 & 433.1 & 570.4 & 391.8 & 462.1 \\
\hline T3 & & 5995.4 & 6540.4 & 5435.3 & 6411.6 & 1590.4 & 1732.9 & 1458.3 & 1708.2 \\
\hline $\mathrm{T} 4$ & & 5698.7 & 5924.7 & 5223.28 & 5902.71 & 1352.9 & 1408.7 & 1310.8 & 1475.1 \\
\hline \multirow[t]{9}{*}{ LSD at 0.05} & & 84.02 & 55.99 & 76.6 & 45.8 & 20.22 & 13.06 & 20.56 & 12.19 \\
\hline & M1 & 5169.4 & 5732.2 & 4878.7 & 5399.9 & 1362.1 & 1502.6 & 1316.3 & 1455.6 \\
\hline & M2 & 5890.7 & 6305.9 & 5624.3 & 5887.1 & 1617.2 & 1717.1 & 1578.0 & 1648.2 \\
\hline & M3 & 5087.5 & 5699.3 & 4651.6 & 5355.6 & 1324.0 & 1471.4 & 1253.7 & 1438.8 \\
\hline & M4 & 5728.6 & 6045.4 & 5520.8 & 5710.6 & 1526.2 & 1610.4 & 1529.4 & 1574.4 \\
\hline & M5 & 4881.8 & 5288.1 & 4486.1 & 5024.0 & 1203.3 & 1297.9 & 1103.8 & 1235.9 \\
\hline & M6 & 4808.0 & 5078.8 & 4209.5 & 4884.3 & 1174.9 & 1235.4 & 1029.2 & 1190.0 \\
\hline & M7 & 4287.1 & 4448.9 & 3830.8 & 4529.9 & 999.0 & 1034.5 & 894.8 & 1054.1 \\
\hline & M8 & 4224.9 & 4460.0 & 3825.9 & 4513.6 & 990.7 & 1045.0 & 899.7 & 1059.4 \\
\hline \multirow[t]{4}{*}{ LSD at 0.05} & & 82.47 & 68.70 & 78.8 & 65.1 & 20.52 & 17.14 & 20.02 & 16.82 \\
\hline & M1 & 6374.4 & 6580.8 & 6321.6 & 6425.3 & 1784.8 & 1842.6 & 1770.0 & 1799.1 \\
\hline & $\mathbf{M} 2$ & 7224.2 & 7358.4 & 6864.0 & 6939.6 & 2095.0 & 2133.9 & 1990.6 & 2012.5 \\
\hline & M3 & 6681.6 & 6716.4 & 6142.80 & 6320.64 & 1870.8 & 1880.6 & 1720.0 & 1769.8 \\
\hline \multirow{8}{*}{ T1 } & M4 & 7004.4 & 6989.5 & 6770.4 & 6706.6 & 1996.3 & 1992.0 & 1929.6 & 1911.4 \\
\hline & M5 & 6310.6 & 6224.6 & 5947.7 & 6119.5 & 1640.7 & 1618.4 & 1546.4 & 1591.1 \\
\hline & M6 & 6363.4 & 5995.2 & 5852.9 & 7.3 & 1654.5 & 8.8 & 1521.7 & 548.9 \\
\hline & M7 & 5660.6 & & & & & & 8.5 & 1405.5 \\
\hline & M8 & 5283.1 & 5883.8 & 5261.3 & 5696.6 & 1320.8 & 1471.0 & 1315.3 & 1424.2 \\
\hline & M1 & 2354.2 & 3045.1 & 2068.8 & 2328.5 & 494.4 & 639.5 & 434.4 & 489.0 \\
\hline & M2 & 2572.8 & 3451.7 & 2491.9 & 2845.4 & 591.7 & 793.9 & 573.1 & 654.5 \\
\hline & M3 & 2107.2 & 2875.7 & 1952.4 & 2507.8 & 463.6 & 632.6 & 429.5 & 551.7 \\
\hline \multirow{8}{*}{$\mathbf{T 2}$} & M4 & 2793.6 & 3169.4 & 2524.8 & 2643.84 & 642.5 & 729.0 & 580.7 & 608.1 \\
\hline & M5 & 1704.0 & 2460.0 & 1574.4 & 1948.3 & 374.9 & 541.2 & 346.4 & 428.6 \\
\hline & M6 & 1622.4 & 2275.2 & 58.4 & 7.2 & 356.9 & 0.5 & 8.8 & 397.6 \\
\hline & M7 & 1339.2 & 4.6 & & & 267.8 & & 7.5 & 290.3 \\
\hline & M8 & 1363.2 & & & & 272.6 & & 243.8 & 277.1 \\
\hline & M1 & 6069.6 & 6753.6 & 5722.1 & 6609.6 & 1699.5 & 1891.0 & 1602.2 & 1850.7 \\
\hline & M2 & 7035.3 & 7579.2 & 6718.3 & 7046.9 & 2065.6 & 2198.0 & 1981.9 & 2078.8 \\
\hline & M3 & 5950.8 & 6854.4 & 5427.8 & 6683.0 & 1586.9 & 1816.4 & 1492.7 & 1837.8 \\
\hline \multirow{8}{*}{ T3 } & M4 & 6458.4 & 7387.2 & 6577.2 & 6950.6 & 1808.4 & 2068.4 & 1874.5 & 1953.1 \\
\hline & M5 & 5984.2 & 6578.2 & 5012.6 & 6296.4 & 1526.0 & 1677.4 & 1278.2 & 1605.6 \\
\hline & M6 & 5888.9 & 6439.0 & 4824.5 & 6140.2 & 1472.2 & 1609.7 & 1206.1 & 1535.0 \\
\hline & M7 & 5238.2 & 5379.1 & 4578.5 & 5826.2 & 1257.2 & 91.0 & 1098.8 & 1398.3 \\
\hline & M8 & 5338.1 & 5352.2 & 4621.2 & 9.6 & 1307.8 & 1311.3 & 1132.2 & 1406.2 \\
\hline & M1 & 5879.5 & 6549.1 & 5402.4 & 6236.2 & 1469.9 & 1637.3 & 1458.7 & 1683.8 \\
\hline & M2 & 6730.6 & 6834.2 & 6423.1 & 6716.4 & 1716.3 & 1742.7 & 1766.4 & 1847.0 \\
\hline & M3 & 5610.2 & 6350.9 & 5083.2 & 5911.0 & 1374.5 & 1556.0 & 1372.5 & 1596.0 \\
\hline \multirow{5}{*}{ T4 } & M4 & 6657.8 & 6635.5 & 6210.7 & 6541.2 & 1657.8 & 1652.2 & 1732.8 & 1825.0 \\
\hline & M5 & 5528.4 & 5889.6 & 5409.6 & 5731.7 & 1271.5 & 1354.6 & 1244.2 & 1318.3 \\
\hline & M6 & 5357.3 & 5605.9 & 4802.4 & 5632.6 & 1216.1 & 1272.5 & 1090.1 & 1278.6 \\
\hline & M7 & 4910.4 & 4725.8 & 4252.8 & & 1055.7 & & 914.4 & 1122.3 \\
\hline & M8 & 4915.2 & 4806.7 & 4201.9 & 5232.7 & 1061.7 & 1038.3 & 907.6 & 1130.3 \\
\hline LSD at 0.05 & & 164.94 & 13740 & 1576 & 1303 & 4104 & 34.27 & 40.03 & 3365 \\
\hline \multicolumn{10}{|c|}{ 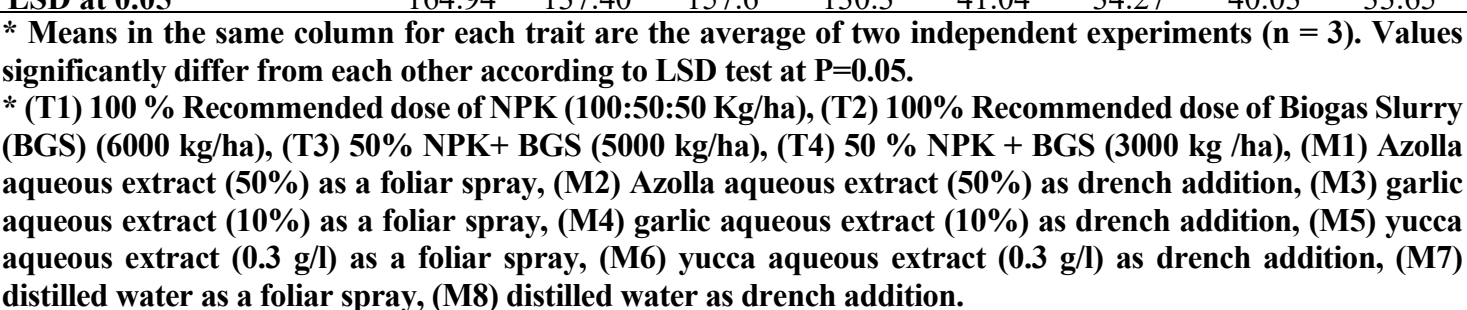 } \\
\hline
\end{tabular}


application methods followed the same pattern previously detected as the recommended dose of chemical fertilizer and Azolla aqueous extract $(50 \%)$ as drench addition $\left(\mathrm{T}_{1} \times \mathrm{M}_{2}\right)$ in the first cut only. The second cut also had the highest levels, in combination with a $50 \% \mathrm{NPK}+5000 \mathrm{~kg} \mathrm{~h}^{-1}$, with Azolla aqueous extract as drench addition $\left(\mathrm{T}_{3} \times \mathrm{M}_{2}\right)$ of both seasons. On the other hand, the interaction of the recommended dose of biogas fertilizer and distilled water as a foliar spray $\left(\mathrm{T}_{2} \times \mathrm{M}_{7}\right)$ in the first cut of both seasons resulted in the lowest fresh and dry weights of leaves Biomass, while the second cut followed the same trend as the first cut but with distilled water as a drench addition $\left(\mathrm{T}_{2} \times \mathrm{M}_{8}\right)$.

\section{Leaf nitrogen percentage (\%):}

Tabulated data in Table (10) revealed that all investigated treatments especially recommended dose of chemical fertilizer $\left(\mathrm{T}_{1}\right)$ recorded the highest nitrogen percentage of both cuts in $1^{\text {st }}$ season as well as $2^{\text {nd }}$ season. Although $50 \% \mathrm{NPK}+5000 \mathrm{~kg} \mathrm{~h}^{-1}$ biogas fertilizer $\left(\mathrm{T}_{3}\right)$ registered the highest nitrogen percentage in the second cut on the second season which had no significant differences with $\left(T_{1}\right)$. In contrast, the lowest values of parameters mentioned above were registered by $100 \%$ recommended dose of biogas fertilizer $\left(\mathrm{T}_{2}\right)$ of both cuts and seasons.

Regarding natural extracts treatments, data state that plants treated with Azolla aqueous extract as drench addition at $50 \%$ $\left(\mathrm{M}_{2}\right)$ gave the highest nitrogen percentage of the two cuts as well as both seasons without significant values with $\left(\mathrm{M}_{4}\right)$ in the first cut only of both seasons while the lowest values were recorded by Azolla aqueous extract $\left(\mathrm{M}_{2}\right)$ of both cuts and seasons.

Additionally, the interaction between recommended dose of chemical fertilizer with garlic aqueous extract $\left(\mathrm{T}_{1} \times \mathrm{M}_{4}\right)$ recorded the highest nitrogen percentage in the first cut only while the second cut of both seasons registered by $50 \% \mathrm{NPK}+5000 \mathrm{~kg} \mathrm{~h}^{-1}$ combining with Azolla aqueous extract
$\left(\mathrm{T}_{3} \times \mathrm{M}_{2}\right)$. On the reverse, the lowest nitrogen content values were recorded by the interaction between a recommended dose of biogas fertilizer and distilled water as a foliar spray as well as drench addition $\left(\mathrm{T}_{2} \times \mathrm{M}_{7}\right)$ without significant differences between them.

\section{Leaf nitrate content:}

According to data presented in Table (10), the recommended dose of chemical fertilizer $\left(T_{1}\right)$ recorded the highest nitrate content in stevia leaf. In contrast, the recommended dose of biogas fertilizer $\left(\mathrm{T}_{2}\right)$ registered the lowest nitrate values in both cuts in both seasons.

Regarding natural extract treatments and their effect on nitrate content, results showed that plants treated with Azolla aqueous extract as drench addition $\left(\mathrm{M}_{2}\right)$ of both cuts and seasons recorded the lowest nitrate content compared to distilled water as a foliar spray $\left(\mathrm{M}_{7}\right)$ as well as distilled water as drench addition $\left(\mathrm{M}_{8}\right)$ without significant differences between them.

Moreover, the effect of the interaction between fertilization and natural extracts showed that the recommended dose of biogas fertilizer with Azolla extract as drench addition $\left(\mathrm{T}_{2} \times \mathrm{M}_{2}\right)$ registered the lowest nitrate values compared to $\mathrm{T}_{1} \times \mathrm{M}_{7}$ treatment which recorded the highest nitrate content in both cuts in both seasons.

\section{Leaf phosphorus and potassium percentage $(\%)$ :}

Concerning the response of the phosphorus and potassium to the different investigated fertilization treatments, data presented in Table (11) show that recommended dose of the chemical fertilizer $\left(\mathrm{T}_{1}\right)$ recorded the highest phosphorus and potassium leaf percentage in the first cut only of both seasons while the second cut of both seasons registered the highest values by using treatment $50 \% \mathrm{NPK}+5000 \mathrm{~kg} \mathrm{~h}^{-1}\left(\mathrm{~T}_{3}\right)$ biogas fertilizer. On the contrary, the lowest values were recorded by recommended dose of biogas fertilizer $\left(\mathrm{T}_{2}\right)$ in the first cut as well as the second cut of both seasons. 
Table 10. Effect of biogas, chemical fertilizer, and natural extracts and their interaction on nitrogen (\%) and nitrate content of Stevia rebaudiana L. plants in 2019 and 2020 seasons for two cuts.

\begin{tabular}{|c|c|c|c|c|c|c|c|c|c|}
\hline \multirow{3}{*}{ Fertilization } & \multirow{3}{*}{$\begin{array}{l}\text { Natural ex. } \\
\text { Methods app }\end{array}$} & \multicolumn{4}{|c|}{$\mathbf{N}(\%)$} & \multicolumn{4}{|c|}{ Nitrate $\mathrm{mg} / \mathrm{kg} \mathrm{dw}$} \\
\hline & & \multicolumn{2}{|c|}{2019} & \multicolumn{2}{|c|}{2020} & \multicolumn{2}{|c|}{2019} & \multicolumn{2}{|c|}{2020} \\
\hline & & $1^{\text {st }}$ cut & $2^{\text {nd }}$ cut & $1^{\text {st }}$ cut & $2^{\text {nd }}$ cut & $1^{\text {st }}$ cut & $2^{\text {nd }}$ cut & $1^{\text {st }}$ cut & $2^{\text {nd }}$ cut \\
\hline T1 & & 1.46 & 1.55 & 1.41 & 1.50 & 25.88 & 28.04 & 27.88 & 28.79 \\
\hline $\mathbf{T 2}$ & & 0.82 & 0.89 & 0.71 & 0.76 & 16.00 & 15.46 & 16.21 & 17.46 \\
\hline T3 & & 1.39 & 1.52 & 1.37 & 1.51 & 21.13 & 25.00 & 22.17 & 27.88 \\
\hline T4 & & 1.27 & 1.38 & 1.27 & 1.39 & 23.29 & 26.00 & 22.79 & 27.50 \\
\hline \multirow[t]{9}{*}{ LSD at 0.05} & & 0.02 & 0.01 & 0.01 & 0.01 & 0.41 & 0.71 & 0.40 & 0.51 \\
\hline & M1 & 1.29 & 1.45 & 1.22 & 1.40 & 20.83 & 23.25 & 21.00 & 25.08 \\
\hline & M2 & 1.42 & 1.52 & 1.35 & 1.50 & 19.50 & 22.50 & 21.00 & 24.17 \\
\hline & M3 & 1.33 & 1.39 & 1.27 & 1.34 & 21.25 & 23.50 & 22.17 & 25.33 \\
\hline & M4 & 1.40 & 1.46 & 1.36 & 1.46 & 21.75 & 23.50 & 21.75 & 25.17 \\
\hline & M5 & 1.22 & 1.34 & 1.14 & 1.26 & 22.58 & 24.25 & 22.42 & 26.08 \\
\hline & M6 & 1.18 & 1.28 & 1.10 & 1.23 & 21.00 & 22.58 & 22.75 & 24.33 \\
\hline & M7 & 1.04 & 1.14 & 1.04 & 1.08 & 23.00 & 24.83 & 23.58 & 26.50 \\
\hline & M8 & 1.02 & 1.13 & 1.04 & 1.06 & 22.67 & 24.58 & 23.42 & 26.58 \\
\hline \multirow[t]{4}{*}{ LSD at 0.05} & & 0.02 & 0.02 & 0.01 & 0.01 & 0.57 & 0.66 & 0.58 & 0.61 \\
\hline & M1 & 1.47 & 1.65 & 1.44 & 1.62 & 25.33 & 27.33 & 25.67 & 28.33 \\
\hline & M2 & 1.66 & 1.73 & 1.54 & 1.65 & 24.00 & 26.00 & 26.00 & 26.67 \\
\hline & M3 & 1.54 & 1.63 & 1.49 & 1.55 & 25.33 & 27.00 & 27.67 & 28.33 \\
\hline \multirow{8}{*}{ T1 } & M4 & 1.71 & 1.65 & 1.63 & 1.68 & 25.67 & 27.67 & 27.33 & 28.33 \\
\hline & M5 & 1.42 & 1.58 & 1.37 & 1.47 & 26.33 & 28.67 & 27.00 & 28.67 \\
\hline & M6 & 1.37 & 1.47 & 1.33 & 1.43 & 25.67 & 26.67 & 28.67 & 28.00 \\
\hline & M7 & 1.29 & 1.40 & 1.25 & 1.31 & 27.33 & 30.67 & 30.33 & 31.00 \\
\hline & M8 & 1.26 & 1.32 & 1.25 & 1.31 & 27.33 & 30.33 & 30.33 & 31.00 \\
\hline & M1 & 0.92 & 1.06 & 0.76 & 0.81 & 16.67 & 16.00 & 14.67 & 16.33 \\
\hline & M2 & 0.96 & 1.10 & 0.85 & 0.96 & 14.33 & 14.67 & 15.67 & 16.00 \\
\hline & M3 & 0.95 & 0.91 & 0.82 & 0.79 & 16.00 & 15.67 & 16.33 & 16.67 \\
\hline \multirow{8}{*}{$\mathbf{T 2}$} & M4 & 0.98 & 0.95 & 0.92 & 0.90 & 16.33 & 16.33 & 16.00 & 17.67 \\
\hline & M5 & 0.83 & 0.93 & 0.63 & 0.74 & 17.33 & 16.00 & 15.67 & 18.67 \\
\hline & M6 & 0.76 & 0.84 & 0.57 & 0.72 & 15.00 & 14.67 & 18.00 & 16.33 \\
\hline & M7 & 0.58 & 0.65 & 0.57 & 0.58 & 16.67 & 15.33 & 16.67 & 18.67 \\
\hline & M8 & 0.57 & 0.68 & 0.56 & 0.57 & 15.67 & 15.00 & 16.67 & 19.33 \\
\hline & M1 & 1.45 & 1.64 & 1.41 & 1.66 & 18.67 & 24.33 & 21.33 & 27.67 \\
\hline & M2 & 1.68 & 1.78 & 1.59 & 1.74 & 18.33 & 24.67 & 20.33 & 27.00 \\
\hline & M3 & 1.51 & 1.57 & 1.49 & 1.57 & 20.33 & 25.67 & 22.00 & 28.67 \\
\hline \multirow{8}{*}{ T3 } & M4 & 1.56 & 1.75 & 1.53 & 1.71 & 21.33 & 24.67 & 21.67 & 28.00 \\
\hline & M5 & 1.36 & 1.46 & 1.32 & 1.45 & 22.67 & 25.67 & 23.33 & 28.67 \\
\hline & M6 & 1.32 & 1.42 & 1.28 & 1.40 & 21.00 & 24.33 & 21.67 & 27.00 \\
\hline & M7 & 1.15 & 1.27 & 1.16 & 1.26 & 23.33 & 25.33 & 23.67 & 28.00 \\
\hline & M8 & 1.14 & 1.27 & 1.16 & 1.26 & 23.33 & 25.33 & 23.33 & 28.00 \\
\hline & M1 & 1.30 & 1.45 & 1.27 & 1.50 & 22.67 & 25.33 & 22.33 & 28.00 \\
\hline & M2 & 1.37 & 1.47 & 1.40 & 1.63 & 21.33 & 24.67 & 22.00 & 27.00 \\
\hline & M3 & 1.33 & 1.45 & 1.30 & 1.47 & 23.33 & 25.67 & 22.67 & 27.67 \\
\hline \multirow{5}{*}{ T4 } & M4 & 1.34 & 1.48 & 1.36 & 1.57 & 23.67 & 25.33 & 22.00 & 26.67 \\
\hline & M5 & 1.27 & 1.37 & 1.23 & 1.37 & 24.00 & 26.67 & 23.67 & 28.33 \\
\hline & M6 & 1.26 & 1.37 & 1.22 & 1.36 & 22.33 & 24.67 & 22.67 & 26.00 \\
\hline & M7 & 1.15 & 1.23 & 1.19 & 1.15 & 24.67 & 28.00 & 23.67 & 28.33 \\
\hline & M8 & 1.13 & 1.25 & 1.18 & 1.11 & 24.33 & 27.67 & 23.33 & 28.00 \\
\hline LSD at 0.05 & & 0.03 & 0.03 & 0.03 & 0.02 & 1.14 & 1.33 & 1.16 & 1.23 \\
\hline
\end{tabular}

* Means in the same column for each trait are the average of two independent experiments $(n=3)$. Values significantly differ from each other according to LSD test at $\mathbf{P}=0.05$.

* (T1) $100 \%$ Recommended dose of NPK (100:50:50 Kg/ha), (T2) 100\% Recommended dose of Biogas Slurry (BGS) (6000 kg/ha), (T3) 50\% NPK+ BGS (5000 kg/ha), (T4) 50 \% NPK + BGS (3000 kg /ha), (M1) Azolla aqueous extract $(50 \%)$ as a foliar spray, (M2) Azolla aqueous extract (50\%) as drench addition, (M3) garlic aqueous extract (10\%) as a foliar spray, (M4) garlic aqueous extract (10\%) as drench addition, (M5) yucca aqueous extract $(0.3 \mathrm{~g} / \mathrm{l})$ as a foliar spray, (M6) yucca aqueous extract (0.3 g/l) as drench addition, (M7) distilled water as a foliar spray, (M8) distilled water as drench addition. 
Table 11. Effect of biogas, chemical fertilizer, and natural extracts and their interaction on phosphorus and potassium content of Stevia rebaudiana L. plants in 2019 and 2020 seasons for two cuts.

\begin{tabular}{|c|c|c|c|c|c|c|c|c|c|}
\hline \multirow{3}{*}{ Fertilization } & \multirow{3}{*}{$\begin{array}{l}\text { Natural ex. } \\
\text { Methods app }\end{array}$} & \multicolumn{4}{|c|}{$P(\%)$} & \multicolumn{4}{|c|}{ K (\%) } \\
\hline & & \multicolumn{2}{|c|}{2019} & \multicolumn{2}{|c|}{2020} & \multicolumn{2}{|c|}{2019} & \multicolumn{2}{|c|}{2020} \\
\hline & & $1^{\text {st }}$ cut & $2^{\text {nd }}$ cut & $1^{\text {st }}$ cut & $2^{\text {nd }}$ cut & $1^{\text {st }}$ cut & $2^{\text {nd }}$ cut & $1^{\text {st }}$ cut & $2^{\text {nd }}$ cut \\
\hline T1 & & 0.212 & 0.220 & 0.204 & 0.216 & 2.329 & 2.377 & 2.386 & 2.407 \\
\hline T2 & & 0.154 & 0.157 & 0.140 & 0.149 & 1.168 & 1.245 & 1.295 & 1.335 \\
\hline T3 & & 0.205 & 0.229 & 0.198 & 0.223 & 2.286 & 2.387 & 2.324 & 2.418 \\
\hline T4 & & 0.171 & 0.211 & 0.180 & 0.213 & 1.745 & 2.238 & 1.757 & 2.276 \\
\hline \multirow[t]{9}{*}{ LSD at 0.05} & & 0.006 & 0.003 & 0.003 & 0.003 & 0.012 & 0.010 & 0.006 & 0.008 \\
\hline & M1 & 0.184 & 0.194 & 0.162 & 0.194 & 1.897 & 2.124 & 1.988 & 2.164 \\
\hline & M2 & 0.202 & 0.221 & 0.198 & 0.220 & 2.073 & 2.182 & 2.056 & 2.228 \\
\hline & M3 & 0.191 & 0.204 & 0.180 & 0.205 & 1.931 & 2.133 & 2.016 & 2.159 \\
\hline & M4 & 0.211 & 0.227 & 0.204 & 0.222 & 2.099 & 2.221 & 2.118 & 2.254 \\
\hline & M5 & 0.189 & 0.212 & 0.189 & 0.211 & 1.846 & 2.052 & 1.899 & 2.107 \\
\hline & M6 & 0.178 & 0.204 & 0.180 & 0.205 & 1.821 & 2.008 & 1.861 & 2.078 \\
\hline & M7 & 0.167 & 0.184 & 0.166 & 0.174 & 1.697 & 1.885 & 1.793 & 1.936 \\
\hline & M8 & 0.165 & 0.186 & 0.165 & 0.172 & 1.693 & 1.888 & 1.795 & 1.947 \\
\hline \multirow[t]{4}{*}{ LSD at 0.05} & & 0.004 & 0.004 & 0.005 & 0.003 & 0.013 & 0.010 & 0.011 & 0.011 \\
\hline & M1 & 0.215 & 0.205 & 0.193 & 0.210 & 2.343 & 2.413 & 2.407 & 2.450 \\
\hline & M2 & 0.225 & 0.244 & 0.220 & 0.236 & 2.487 & 2.453 & 2.483 & 2.487 \\
\hline & M3 & 0.213 & 0.216 & 0.197 & 0.220 & 2.360 & 2.427 & 2.437 & 2.433 \\
\hline \multirow{8}{*}{ T1 } & M4 & 0.235 & 0.238 & 0.227 & 0.244 & 2.510 & 2.480 & 2.517 & 2.527 \\
\hline & M5 & 0.218 & 0.236 & 0.215 & 0.227 & 2.290 & 2.367 & 2.370 & 2.393 \\
\hline & M6 & 0.206 & 0.226 & 0.210 & 0.225 & 2.277 & 2.333 & 2.337 & 2.347 \\
\hline & M7 & 0.194 & 0.196 & 0.188 & 0.186 & 2.197 & 2.270 & 2.267 & 2.307 \\
\hline & M8 & 0.192 & 0.195 & 0.185 & 0.183 & 2.170 & 2.273 & 2.273 & 2.310 \\
\hline & M1 & 0.150 & 0.161 & 0.139 & 0.146 & 1.217 & 1.260 & 1.320 & 1.357 \\
\hline & M2 & 0.183 & 0.164 & 0.154 & 0.165 & 1.240 & 1.320 & 1.380 & 1.410 \\
\hline & M3 & 0.163 & 0.169 & 0.141 & 0.155 & 1.223 & 1.297 & 1.337 & 1.380 \\
\hline \multirow{8}{*}{$\mathbf{T} 2$} & M4 & 0.173 & 0.180 & 0.156 & 0.164 & 1.260 & 1.370 & 1.420 & 1.437 \\
\hline & M5 & 0.151 & 0.155 & 0.145 & 0.148 & 1.190 & 1.240 & 1.270 & 1.323 \\
\hline & M6 & 0.145 & 0.145 & 0.130 & 0.143 & 1.160 & 1.220 & 1.243 & 1.290 \\
\hline & M7 & 0.134 & 0.137 & 0.127 & 0.137 & 1.023 & 1.127 & 1.190 & 1.227 \\
\hline & M8 & 0.135 & 0.144 & 0.126 & 0.134 & 1.030 & 1.128 & 1.197 & 1.260 \\
\hline & M1 & 0.203 & 0.216 & 0.163 & 0.215 & 2.290 & 2.443 & 2.353 & 2.443 \\
\hline & M2 & 0.207 & 0.252 & 0.215 & 0.244 & 2.463 & 2.543 & 2.417 & 2.563 \\
\hline & M3 & 0.203 & 0.224 & 0.193 & 0.228 & 2.323 & 2.423 & 2.383 & 2.407 \\
\hline \multirow{8}{*}{ T3 } & M4 & 0.233 & 0.257 & 0.222 & 0.247 & 2.477 & 2.563 & 2.473 & 2.573 \\
\hline & M5 & 0.214 & 0.231 & 0.207 & 0.237 & 2.247 & 2.370 & 2.317 & 2.380 \\
\hline & M6 & 0.204 & 0.223 & 0.203 & 0.229 & 2.220 & 2.340 & 2.260 & 2.360 \\
\hline & M7 & 0.191 & 0.215 & 0.193 & 0.193 & 2.133 & 2.203 & 2.197 & 2.310 \\
\hline & M8 & 0.188 & 0.213 & 0.185 & 0.192 & 2.137 & 2.207 & 2.195 & 2.310 \\
\hline & M1 & 0.167 & 0.195 & 0.153 & 0.204 & 1.737 & 2.380 & 1.870 & 2.407 \\
\hline & M2 & 0.193 & 0.225 & 0.203 & 0.235 & 2.100 & 2.410 & 1.943 & 2.450 \\
\hline & M3 & 0.183 & 0.207 & 0.191 & 0.217 & 1.817 & 2.387 & 1.907 & 2.417 \\
\hline \multirow{5}{*}{ T4 } & M4 & 0.203 & 0.233 & 0.211 & 0.234 & 2.150 & 2.470 & 2.060 & 2.480 \\
\hline & M5 & 0.173 & 0.226 & 0.187 & 0.234 & 1.657 & 2.230 & 1.640 & 2.330 \\
\hline & M6 & 0.157 & 0.220 & 0.177 & 0.224 & 1.627 & 2.140 & 1.603 & 2.317 \\
\hline & M7 & 0.147 & 0.190 & 0.157 & 0.179 & 1.433 & 1.940 & 1.517 & 1.900 \\
\hline & M8 & 0.143 & 0.194 & 0.163 & 0.178 & 1.437 & 1.943 & 1.513 & 1.907 \\
\hline LSD at 0.05 & & 0.008 & 0.007 & 0.009 & 0.006 & 0.026 & 0.020 & 0.022 & 0.023 \\
\hline
\end{tabular}

* Means in the same column for each trait are the average of two independent experiments $(n=3)$. Values significantly differ from each other according to LSD test at $\mathbf{P}=\mathbf{0 . 0 5}$.

* (T1) $100 \%$ Recommended dose of NPK (100:50:50 Kg/ha), (T2) 100\% Recommended dose of Biogas Slurry (BGS) (6000 kg/ha), (T3) 50\% NPK+ BGS (5000 kg/ha), (T4) $50 \%$ NPK + BGS (3000 kg /ha), (M1) Azolla aqueous extract (50\%) as a foliar spray, (M2) Azolla aqueous extract (50\%) as drench addition, (M3) garlic aqueous extract (10\%) as a foliar spray, (M4) garlic aqueous extract (10\%) as drench addition, (M5) yucca aqueous extract $(0.3 \mathrm{~g} / \mathrm{l})$ as a foliar spray, (M6) yucca aqueous extract $(0.3 \mathrm{~g} / \mathrm{l})$ as drench addition, (M7) distilled water as a foliar spray, (M8) distilled water as drench addition. 
Referring to the response of phosphorus and potassium to natural extracts data presented in Table (11) display obviously that garlic extract $\left(\mathrm{M}_{4}\right)$ recorded the highest values of both cuts in the first and second cuts meanwhile the lowest values were recorded by distilled water as foliar spray or drench addition $\left(\mathrm{T}_{7}\right.$ or $\left.\mathrm{T}_{8}\right)$ without significant difference between them.

As for the interaction between the two factors, the results presented in Table (11) revealed that the interaction between recommended dose of chemical fertilizer and garlic aqueous extract as drench addition $\left(\mathrm{T}_{1} \times \mathrm{M}_{4}\right)$ registered the highest phosphorus and potassium leave percentage in the first cut only of both seasons. On the other hand, the interaction between $50 \% \mathrm{NPK}+5000 \mathrm{~kg}$ $\mathrm{h}^{-1}$ biogas fertilizer and garlic aqueous extract as drench addition $\left(\mathrm{T}_{3} \times \mathrm{M}_{4}\right)$ recorded the highest values in the second cut of both seasons. Meanwhile, the lowest values were recorded by the interaction between a recommended dose of biogas fertilizer and distilled water as a foliar spray $\left(\mathrm{T}_{2} \times \mathrm{M}_{7}\right)$ as well as drench addition $\left(\mathrm{T}_{2} \times \mathrm{M}_{8}\right)$ without significant differences between them.

\section{Chlorophyll a and chlorophyll b content:}

Data presented in Table (12) display obviously that investigated treatments with any biogas fertilizer and natural extracts and their interaction significantly increased the chlorophyll content. As for $50 \% \mathrm{NPK}+5000$ $\mathrm{kg} \mathrm{h}^{-1}$ biogas fertilizer $\left(\mathrm{T}_{3}\right)$ recorded the highest chlorophyll $\mathrm{a}$ and $\mathrm{b}$ content in the first and second cuts of both seasons. In contrast, the lowest chlorophyll a and $b$ content values were recorded by recommended dose of biogas fertilizer $\left(\mathrm{T}_{2}\right)$.

Concerning natural extracts the highest content of chlorophyll a and b contents were recorded by Azolla aqueous extract as drench addition $\left(\mathrm{M}_{2}\right)$ of both cuts in the first and second seasons while the lowest chlorophyll $\mathrm{a}$ and $\mathrm{b}$ contents were registered by distilled water $\left(\mathrm{M}_{7}\right)$ as well as foliar or drench addition $\left(\mathrm{M}_{8}\right)$ without significant variation between them.
On the other side, the interaction between the recommended dose of chemical fertilizer and Azolla aqueous extract as drench addition $\left(\mathrm{T}_{1} \times \mathrm{M}_{2}\right)$ registered the highest chlorophyll $a$ and $b$ in the first cut of both seasons while in the second cut of both seasons the highest values were recorded by the combination between $50 \%$ NPK +5000 $\mathrm{kg} \mathrm{h}^{-1}$ with Azolla aqueous extract as drench addition $\left(\mathrm{T}_{3} \times \mathrm{M}_{2}\right)$. However, the minimum values in chlorophyll $\mathrm{a}$ and $\mathrm{b}$ registered coming from the interaction between a recommended dose of biogas fertilizer $\left(\mathrm{T}_{2}\right)$ with distilled water as foliar as well as drench addition without significant variation between them.

\section{Carotenoids and total chlorophylls content:}

Data presented Table (13) show obviously the response of carotenoids and total chlorophylls to different fertilization rates of recommended dose of chemical fertilizer $\left(T_{1}\right)$ in the first cut only in the first and second seasons recorded the highest values of the carotenoids content while the second cut of both seasons registered the highest values by $50 \%$ NPK $+5000 \mathrm{~kg} \mathrm{~h}^{-1}$ biogas fertilizer $\left(\mathrm{T}_{3}\right)$, likewise total chlorophyll which took the same trend with the second cut of carotenoids content of both cuts and seasons. On the contrary, the lowest values of carotenoids, as well as total chlorophylls, were recorded by the recommended dose of biogas fertilizer of both cuts and seasons.

Regarding natural extracts application methods, the highest values of both carotenoids and total chlorophylls were recorded by Azolla aqueous extract as drench addition while the lowest values were recorded by distilled water as foliar or drench addition without significant differences between them.

On the other hand, the interaction between of recommended dose of chemical fertilizer with Azolla aqueous extract as drench addition $\left(\mathrm{T}_{1} \times \mathrm{M}_{2}\right)$ in the first cut only recorded the highest carotenoids and total 
Table 12. Effect of biogas, chemical fertilizer, and natural extracts and their interaction on chlorophyll a and chlorophyll b of Stevia rebaudiana L. plants in 2019 and 2020 seasons for two cuts.

\begin{tabular}{|c|c|c|c|c|c|c|c|c|c|}
\hline \multirow{3}{*}{ Fertilization } & \multirow{3}{*}{$\begin{array}{l}\text { Natural ex. } \\
\text { Methods app }\end{array}$} & \multicolumn{4}{|c|}{ Chl a (mg g-1 fw) } & \multicolumn{4}{|c|}{ Chl b (mg g-1 fw) } \\
\hline & & \multicolumn{2}{|c|}{2019} & \multicolumn{2}{|c|}{2020} & \multicolumn{2}{|c|}{2019} & \multicolumn{2}{|c|}{2020} \\
\hline & & $1^{\text {st }}$ cut & $2^{\text {nd }}$ cut & $1^{\text {st }}$ cut & $2^{\text {nd }}$ cut & $1^{\text {st }}$ cut & $2^{\text {nd }}$ cut & $1^{\text {st }}$ cut & $2^{\text {nd }}$ cut \\
\hline T1 & & 0.3572 & 0.3552 & 0.3656 & 0.3628 & 0.1141 & 0.1184 & 0.1179 & 0.1209 \\
\hline $\mathbf{T 2}$ & & 0.3121 & 0.3214 & 0.3228 & 0.3284 & 0.1038 & 0.1071 & 0.1077 & 0.1094 \\
\hline T3 & & 0.3555 & 0.3744 & 0.3673 & 0.3823 & 0.1186 & 0.1238 & 0.1227 & 0.1276 \\
\hline T4 & & 0.3330 & 0.3415 & 0.3397 & 0.3525 & 0.1118 & 0.1138 & 0.1157 & 0.1175 \\
\hline \multirow[t]{9}{*}{ LSD at 0.05} & & 0.0045 & 0.0017 & 0.0027 & 0.0029 & 0.0007 & 0.0007 & 0.0005 & 0.0009 \\
\hline & M1 & 0.3464 & 0.3598 & 0.3548 & 0.3678 & 0.1155 & 0.1199 & 0.1193 & 0.1226 \\
\hline & M2 & 0.4102 & 0.4255 & 0.4204 & 0.4337 & 0.1367 & 0.1418 & 0.1401 & 0.1446 \\
\hline & M3 & 0.3559 & 0.3669 & 0.3675 & 0.3771 & 0.1186 & 0.1223 & 0.1221 & 0.1257 \\
\hline & M4 & 0.3985 & 0.4162 & 0.4134 & 0.4246 & 0.1342 & 0.1376 & 0.1384 & 0.1415 \\
\hline & M5 & 0.3684 & 0.3428 & 0.3679 & 0.3524 & 0.1148 & 0.1143 & 0.1197 & 0.1175 \\
\hline & M6 & 0.3408 & 0.3314 & 0.3493 & 0.3563 & 0.1127 & 0.1105 & 0.1164 & 0.1188 \\
\hline & M7 & 0.2473 & 0.2705 & 0.2593 & 0.2692 & 0.0826 & 0.0902 & 0.0860 & 0.0898 \\
\hline & M8 & 0.2483 & 0.2718 & 0.2580 & 0.2709 & 0.0817 & 0.0897 & 0.0860 & 0.0906 \\
\hline \multirow[t]{4}{*}{ LSD at 0.05} & & 0.0044 & 0.0030 & 0.0036 & 0.0024 & 0.0010 & 0.0011 & 0.0007 & 0.0008 \\
\hline & M1 & 0.3677 & 0.3667 & 0.3753 & 0.3713 & 0.1226 & 0.1222 & 0.1251 & 0.1238 \\
\hline & M2 & 0.4290 & 0.4410 & 0.4390 & 0.4483 & 0.1430 & & & 494 \\
\hline & M3 & 0.3847 & 0.3813 & 0.3957 & 0.4029 & 0.1282 & 0.1271 & & 0.1343 \\
\hline \multirow{8}{*}{$\mathbf{T 1}$} & M4 & 0.4250 & 0.4290 & 0.4333 & & 0.1417 & 0.14 & 444 & 478 \\
\hline & M5 & 0.4070 & 0.3347 & 0.4210 & 0.3447 & 0.1038 & 0.11 & 0.1084 & 0.1149 \\
\hline & M6 & 0.3273 & 0.3250 & 0.3313 & 0.3420 & 0.1056 & 0.1083 & 0.1104 & 0.1140 \\
\hline & M7 & 0.2580 & 0.2817 & 0.2643 & 0.2727 & 0.0860 & 0.0939 & 0.0881 & 0.0909 \\
\hline & M8 & 0.2590 & 0.2820 & 0.2650 & 0.2773 & 0.0821 & 0.0940 & 0.0883 & 0.0924 \\
\hline & M1 & 0.3183 & 0.3343 & 0.3167 & 0.3400 & 0.1061 & 0.1114 & 0.1097 & 0.1133 \\
\hline & M2 & 0.3830 & 0.3920 & 0.3930 & 0.4017 & 0.1277 & 0.1307 & 0.1310 & 0.1339 \\
\hline & M3 & 0.3280 & 0.3 & 117 & 0.3370 & 0.1093 & & 0.1 & 0.1123 \\
\hline \multirow{8}{*}{$\mathbf{T} 2$} & M4 & 0.3720 & 0.379 & 0.3850 & 0.3 & 0.1240 & 0.1 & 0.1 & 0.1281 \\
\hline & M5 & 0.3180 & 0.324 & 0.3343 & 0.3300 & 0.1060 & & & 100 \\
\hline & M6 & 0.3070 & 0.3133 & 0.3187 & 0.3250 & 0.1023 & 0.1044 & 0.1062 & 0.1083 \\
\hline & M7 & 0.2354 & 0.2430 & 0.2487 & 0.2539 & 0.0785 & 0.0810 & 0.0811 & 0.0843 \\
\hline & M8 & 0.2353 & 0.2450 & 0.2443 & 0.2550 & 0.0769 & 0.0817 & 0.0814 & 0.0850 \\
\hline & M1 & 0.3590 & 0.3870 & 0.3763 & 0.4017 & 0.1197 & 0.1290 & 0.1254 & 0.1339 \\
\hline & M2 & 0.4257 & 0.4573 & 0.4357 & 0.4610 & 0.1419 & 0.1524 & 0.1452 & 0.1537 \\
\hline & M3 & 0.3640 & 0.3940 & 0.3757 & 0.4043 & 0.1213 & 0.1313 & 0.1252 & 0.1348 \\
\hline \multirow{8}{*}{ T3 } & M4 & 0.4183 & 0.4533 & 0.4250 & 0.4507 & 0.1394 & 0.1467 & 0.1439 & 0.1502 \\
\hline & M5 & 0.3873 & 0.3487 & 0.3997 & 0.3587 & 0.1291 & 0.1162 & 0.1332 & 0.1196 \\
\hline & M6 & 0.3833 & 0.3357 & 0.3933 & 0.3953 & 0.1278 & 0.1119 & 0.1311 & 0.1318 \\
\hline & M7 & 0.2530 & 0.3090 & 0.2683 & 0.2930 & 0.0850 & 0.1030 & 0.0894 & 0.0977 \\
\hline & M8 & 0.2537 & 0.3103 & 0.2640 & 0.2940 & 0.0846 & 0.0997 & 0.0880 & 0.0989 \\
\hline & M1 & 0.3407 & 0.3510 & 0.3510 & 0.3583 & 0.1136 & 0.1170 & 0.1170 & 0.1194 \\
\hline & M2 & 0.4030 & 0.4117 & 0.4140 & 0.4237 & 0.1343 & 0.1372 & 0.1380 & 0.1412 \\
\hline & M3 & 0.3470 & 0.3533 & 0.3570 & 0.3640 & 0.1157 & 0.1178 & 0.1190 & 0.1213 \\
\hline \multirow{5}{*}{ T4 } & M4 & 0.3787 & 0.4027 & 0.4104 & 0.4200 & 0.1318 & 0.1342 & 0.1368 & 0.1400 \\
\hline & M5 & 0.3613 & 0.3630 & 0.3167 & 0.3763 & 0.1204 & 0.1210 & 0.1256 & 0.1254 \\
\hline & M6 & 0.3453 & 0.3517 & 0.3540 & 0.3630 & 0.1151 & 0.1172 & 0.1180 & 0.1210 \\
\hline & M7 & 0.2430 & 0.2483 & 0.2557 & 0.2573 & 0.0810 & 0.0828 & 0.0852 & 0.0857 \\
\hline & M8 & 0.2450 & 0.2500 & 0.2587 & 0.2573 & 0.0824 & 0.0833 & 0.0862 & 0.0861 \\
\hline LSD at 0.05 & & 0.0088 & 0.0059 & 0.0071 & 0.0048 & 0.0019 & 0.0023 & 0.0015 & 0.0016 \\
\hline \multicolumn{10}{|c|}{$\begin{array}{l}\text { * Means in the same column for each trait are the average of two independent experiments }(\mathrm{n}=3) \text {. Values } \\
\text { significantly differ from each other according to LSD test at } \mathrm{P}=0.05 \text {. } \\
\text { * (T1) } 100 \% \text { Recommended dose of NPK }(100: 50: 50 \mathrm{Kg} / \mathrm{ha}),(\mathrm{T} 2) 100 \% \text { Recommended dose of Biogas Slurry } \\
\text { (BGS) }(6000 \mathrm{~kg} / \mathrm{ha}),(\mathrm{T} 3) 50 \% \text { NPK+ BGS }(5000 \mathrm{~kg} / \mathrm{ha}),(\mathrm{T} 4) 50 \% \mathrm{NPK}+\mathrm{BGS}(3000 \mathrm{~kg} / \mathrm{ha}) \text {, (M1) Azolla } \\
\text { aqueous extract }(50 \%) \text { as a foliar spray, (M2) Azolla aqueous extract }(50 \%) \text { as drench addition, (M3) garlic } \\
\text { aqueous extract }(10 \%) \text { as a foliar spray, (M4) garlic aqueous extract }(10 \%) \text { as drench addition, (M5) yucca } \\
\text { aqueous extract }(0.3 \mathrm{~g} / \mathrm{l}) \text { as a foliar spray, (M6) yucca aqueous extract }(0.3 \mathrm{~g} / \mathrm{l}) \text { as drench addition, (M7) } \\
\text { distilled water as a foliar spray, (M8) distilled water as drench addition. }\end{array}$} \\
\hline
\end{tabular}


Table 13. Effect of biogas, chemical fertilizer, and natural extracts and their interaction on carotenoids and Total chlorophylls of Stevia rebaudiana L. plants in 2019 and 2020 seasons for two cuts.

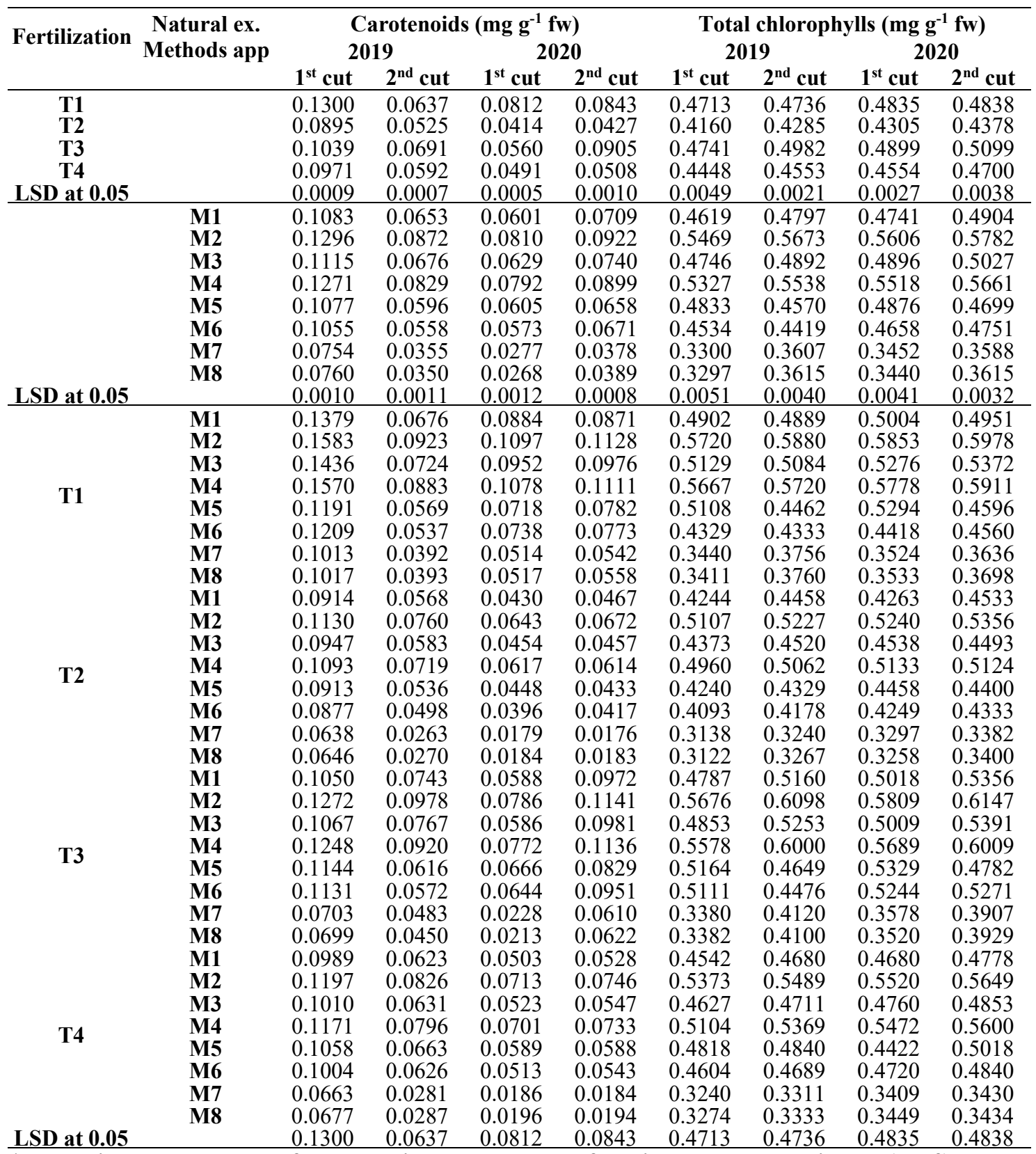

* Means in the same column for each trait are the average of two independent experiments $(n=3)$. Values significantly differ from each other according to LSD test at $\mathbf{P}=\mathbf{0 . 0 5}$.

* (T1) $100 \%$ Recommended dose of NPK (100:50:50 Kg/ha), (T2) 100\% Recommended dose of Biogas Slurry (BGS) (6000 kg/ha), (T3) 50\% NPK+ BGS (5000 kg/ha), (T4) 50 \% NPK + BGS (3000 kg /ha), (M1) Azolla aqueous extract (50\%) as a foliar spray, (M2) Azolla aqueous extract (50\%) as drench addition, (M3) garlic aqueous extract $(\mathbf{1 0 \%})$ as a foliar spray, (M4) garlic aqueous extract (10\%) as drench addition, (M5) yucca aqueous extract $(0.3 \mathrm{~g} / \mathrm{l})$ as a foliar spray, (M6) yucca aqueous extract (0.3 g/l) as drench addition, (M7) distilled water as a foliar spray, (M8) distilled water as drench addition. 
chlorophylls content of both seasons. Even though the second cut of both seasons took the same line with the first cut whereas Azolla aqueous extract used as drench addition with $50 \%$ NPK $+5000 \mathrm{~kg} \mathrm{~h}^{-1}$ biogas fertilizer $\left(\mathrm{T}_{3} \times \mathrm{M}_{2}\right)$. In contrast, the lowest values of carotenoids along with total chlorophylls were registered by recommended dose of biogas fertilizer with distilled water as a foliar spray $\left(\mathrm{T}_{2} \times \mathrm{M}_{7}\right)$ of both cuts and seasons.

\section{Stevioside and rebaudioside A., content:}

Stevioside and rebaudioside A., (steviol glycoside) are the main glycosides that are responsible for the quality of stevia. Their concentration in the plant determines the sweetness and bitterness. A perusal of data presented in Table (14) and Figs. (1-4) shows that biogas fertilizer as organic manure and natural extracts of stevia recorded a significant effect on stevioside concentration as the highest values of stevioside was recorded by $50 \% \mathrm{NPK}+3000$ $\mathrm{kg} \mathrm{h}{ }^{-1}$ combining with Azolla aqueous extract as drench addition $\left(\mathrm{T}_{4} \times \mathrm{M}_{2}\right)$ followed by $50 \% \mathrm{NPK}+3000 \mathrm{~kg} \mathrm{~h}^{-1}\left(\mathrm{~T}_{4} \mathrm{M}_{6}\right)$ as well as recommended dose of chemical fertilizer with Azolla aqueous extract as a drench addition $\left(\mathrm{T}_{1} \times \mathrm{M}_{2}\right)$.

On the other hand, the highest value of both total steviol glycoside (stevioside and rebaudioside A., was recorded by $50 \%$ $\mathrm{NPK}+3000 \mathrm{~kg} \mathrm{~h}^{-1}$ combining with Azolla aqueous extract as drench addition $\left(\mathrm{T}_{4} \times \mathrm{M}_{2}\right)$ followed by recommended dose of chemical fertilizer with Azolla aqueous extract as a drench addition $\left(\mathrm{T}_{1} \times \mathrm{M}_{2}\right)$ while the interaction treatments of $50 \% \mathrm{NPK}+\mathrm{BGS}$ $\left(5000 \mathrm{~kg} \mathrm{ha}^{-1}\right)+$ Azolla extract as drench addition $\left(\mathrm{T}_{3} \times \mathrm{M}_{2}\right)$ ranked the third in this concern.

Meanwhile, as data presented in the same Table showed that rebaudioside A., registered the highest values with the interaction between the recommended dose of biogas fertilizer and yucca aqueous extract as a foliar spray $\left(\mathrm{T}_{2} \times \mathrm{M}_{5}\right)$ followed by the interaction between the recommended dose of biogas fertilizer and Azolla aqueous extract as a drench addition $\left(\mathrm{T}_{2} \times \mathrm{M}_{2}\right)$ followed by the interaction between the recommended dose of biogas fertilizer and Azolla aqueous extract as a drench addition $\left(\mathrm{T}_{2} \times \mathrm{M}_{4}\right)$.

\section{DISCUSSION}

Biogas-slurry is a great soil conditioner because it adds humus and increased the organic matter, available phosphorus, and exchangeable potassium content of the soil, as well as its porosity and water holding capacity. It also reduces soil temperature fluctuations, decreases soil water evaporation, and influence on the levels of certain nutrients measured in plants (Mdlambuzi et al., 2021). Chemical fertilizers add more nutrients to the soil than organic fertilizers, but they can only deliver certain nutrients to the crops without enhancing soil production. However chemical fertilizers are prohibitively expensive for small-scale farmers. (Yamika et al., 2019) Moreover, biogas slurry lowers the cost of natural organic fertilizer while increasing soil fertility Because the physical conditions of the soil have been altered, nutrient uptake by plants has increased, resulting in increased crop yield. (Yan et al., 2021)

The reason for plant's shoots fresh and dry yield grows and gave the highest values only for the first cut as a result of the use of yucca aqueous extract as a foliar spray with chemical fertilizer, as yucca extract works to break the surface tension of the leaf because it contains steroidal saponins, allowing for more efficient gas exchange, which increases the plant's ability to perform photosynthesis and water and food absorption (Andreuccetti et al., 2011). This study's finding is coincided with those reported by (Jonas, 1969), who found that the application of aqueous saponin-rich extracts of Digitalis purpurea stimulated the formation of adventitious roots on tomato cuttings, whereas application of the pure saponin, digitoxin, had the opposite effect, suggesting that digitoxin is toxic to tomato plants. 
Table 14. LC-MS chromatograms of Stevia rebaudiana $L$. leaves in the first cut of the second season (2020).

\begin{tabular}{|c|c|c|c|}
\hline Treatments & Stev.* & $\operatorname{Reb} \mathbf{A}^{* *}$ & $\begin{array}{c}\text { Total } \\
\text { Stev. + } \\
\text { RebA } \\
\end{array}$ \\
\hline T1M1: $100 \%$ RD of NPK +Azolla ex.. as a foliar spray & 9.53 & 0.13 & 9.66 \\
\hline T1M2: $100 \%$ RD of NPK+Azolla ex. as drench addition & 9.76 & 0.27 & 10.03 \\
\hline T1M3: $100 \%$ RD of NPK+ garlic ex. as a foliar spray & 9.35 & 0.28 & 9.63 \\
\hline T1M4: $100 \%$ RD of NPK + garlic ex. as drench addition & 9.45 & 0.11 & 9.56 \\
\hline T1M5: $100 \%$ RD of NPK+ yucca ex. as a foliar spray & 9.25 & 0.20 & 9.45 \\
\hline $\mathrm{T}_{1} \mathrm{M} 6: 100 \% \mathrm{RD}$ of NPK+ yucca ex. as drench addition & 9.62 & 0.12 & 9.74 \\
\hline T1M7: $100 \%$ RD of NPK+ distilled water as a foliar spray & 9.18 & 0.18 & 9.36 \\
\hline T1M8: $100 \% \mathrm{RD}$ of NPK + distilled water as drench addition & 9.12 & 0.09 & 9.21 \\
\hline T2M1: $100 \%$ RD of BGS $(6000 \mathrm{~kg} / \mathrm{ha})+$ Azolla extract as a foliar spray & 4.22 & 1.10 & 5.32 \\
\hline T2M2: $100 \%$ RD of BGS $(6000 \mathrm{~kg} / \mathrm{ha})+$ Azolla extract as drench addition & 4.56 & 2.00 & 6.56 \\
\hline T2M3: $100 \%$ RD of BGS (6000 kg/ha) + garlic extract as a foliar spray & 2.71 & 0.54 & 3.25 \\
\hline T2M4: $100 \%$ RD of BGS $(6000 \mathrm{~kg} / \mathrm{ha})+$ garlic extract as drench addition & 1.75 & 1.65 & 3.40 \\
\hline T2M5: $100 \%$ RD of BGS (6000 kg/ha) + yucca extract as foliar spray & 2.04 & 2.79 & 4.83 \\
\hline T2M6: $100 \%$ RD of BGS (6000 kg/ha) +yucca extract as drench addition & 2.28 & 1.46 & 3.73 \\
\hline T2M7: $100 \% \mathrm{RD}$ of BGS $(6000 \mathrm{~kg} / \mathrm{ha})+$ distilled water as foliar spray & 1.93 & 0.41 & 2.34 \\
\hline T2M8: $100 \%$ RD of BGS $(6000 \mathrm{~kg} / \mathrm{ha})+$ distilled water as drench addition & 2.02 & 0.45 & 2.47 \\
\hline T3M1: 50\% NPK+ BGS (5000 kg/ha) +Azolla extract as foliar spray & 4.59 & 0.51 & 5.09 \\
\hline T3M2: $50 \% \mathrm{NPK}+\mathrm{BGS}(5000 \mathrm{~kg} / \mathrm{ha})+$ Azolla extract as drench addition & 9.58 & 0.34 & 9.92 \\
\hline T3M3: $50 \% \mathrm{NPK}+\mathrm{BGS}(5000 \mathrm{~kg} / \mathrm{ha})+$ garlic extract as foliar spray & 6.12 & 1.36 & 7.48 \\
\hline T3M4: $50 \% \mathrm{NPK}+\mathrm{BGS}(5000 \mathrm{~kg} / \mathrm{ha})+$ garlic extract as drench addition & 9.10 & 0.78 & 9.89 \\
\hline T3M5: 50\% NPK+ BGS (5000 kg/ha) + yucca extract as foliar spray & 6.64 & 0.06 & 6.70 \\
\hline T3M6: $50 \% \mathrm{NPK}+\mathrm{BGS}(5000 \mathrm{~kg} / \mathrm{ha})+$ yucca extract as drench addition & 9.32 & 0.57 & 9.89 \\
\hline T3M7: 50\% NPK+ BGS (5000 kg/ha) + distilled water as foliar spray & 4.12 & 0.11 & 4.23 \\
\hline T3M8: $50 \% \mathrm{NPK}+\mathrm{BGS}(5000 \mathrm{~kg} / \mathrm{ha})+$ distilled water as drench addition & 4.12 & 0.11 & 4.23 \\
\hline T4M1: $50 \% \mathrm{NPK}+\mathrm{BGS}(3000 \mathrm{~kg} / \mathrm{ha})+$ Azolla extract as foliar spray & 9.43 & 0.10 & 9.53 \\
\hline T4M2: $50 \% \mathrm{NPK}+\mathrm{BGS}(3000 \mathrm{~kg} / \mathrm{ha})+$ Azolla extract as drench addition & 9.83 & 0.54 & 10.37 \\
\hline T4M3: 50\% NPK+ BGS (3000 kg/ha) + garlic extract as foliar spray & 9.09 & 0.75 & 9.84 \\
\hline T4M4: 50\% NPK+ BGS (3000 kg/ha) + garlic extract as drench addition & 9.49 & 0.13 & 9.62 \\
\hline T4M5: 50\% NPK+ BGS (3000 kg/ha) + yucca extract as foliar spray & 8.57 & 0.22 & 8.79 \\
\hline T4M6: $50 \% \mathrm{NPK}+\mathrm{BGS}(3000 \mathrm{~kg} / \mathrm{ha})+$ yucca extract as drench addition & 9.76 & 0.12 & 9.88 \\
\hline T4M7: 50\% NPK+ BGS (3000 kg/ha) + distilled water as foliar spray & 6.72 & 0.06 & 6.78 \\
\hline T4M8: $50 \% \mathrm{NPK}+\mathrm{BGS}(3000 \mathrm{~kg} / \mathrm{ha})+$ distilled water as drench addition & 6.95 & 0.09 & 7.04 \\
\hline
\end{tabular}

*Stevioside

${ }^{* *}$ Rebaudioside A. 


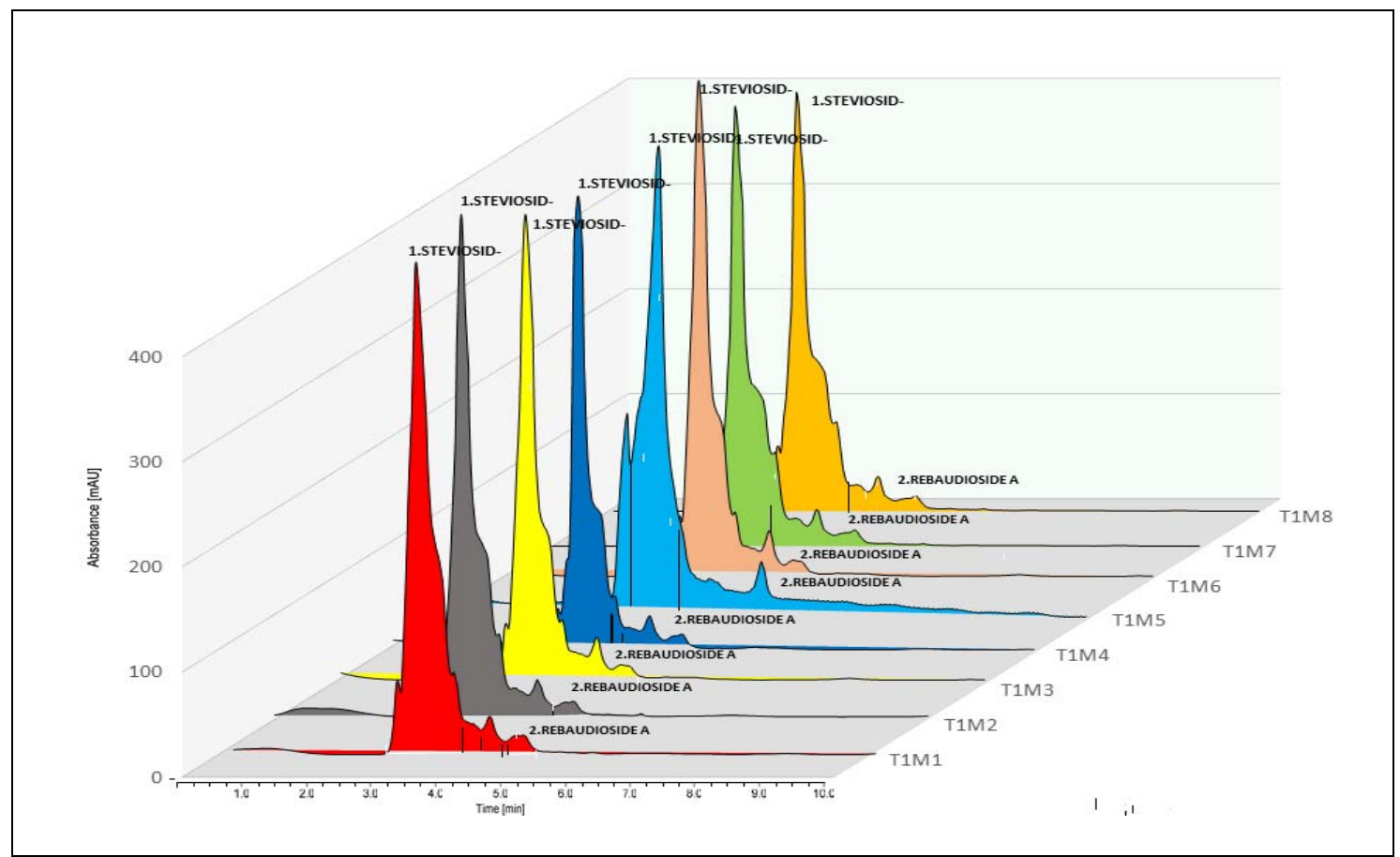

Fig. 1. LC-MS the effect of chemical fertilizer and natural extracts method application on Stevioside and rebaudioside A., chromatogram of Stevia rebaudiana leaves in the first cut of the second season (2020).

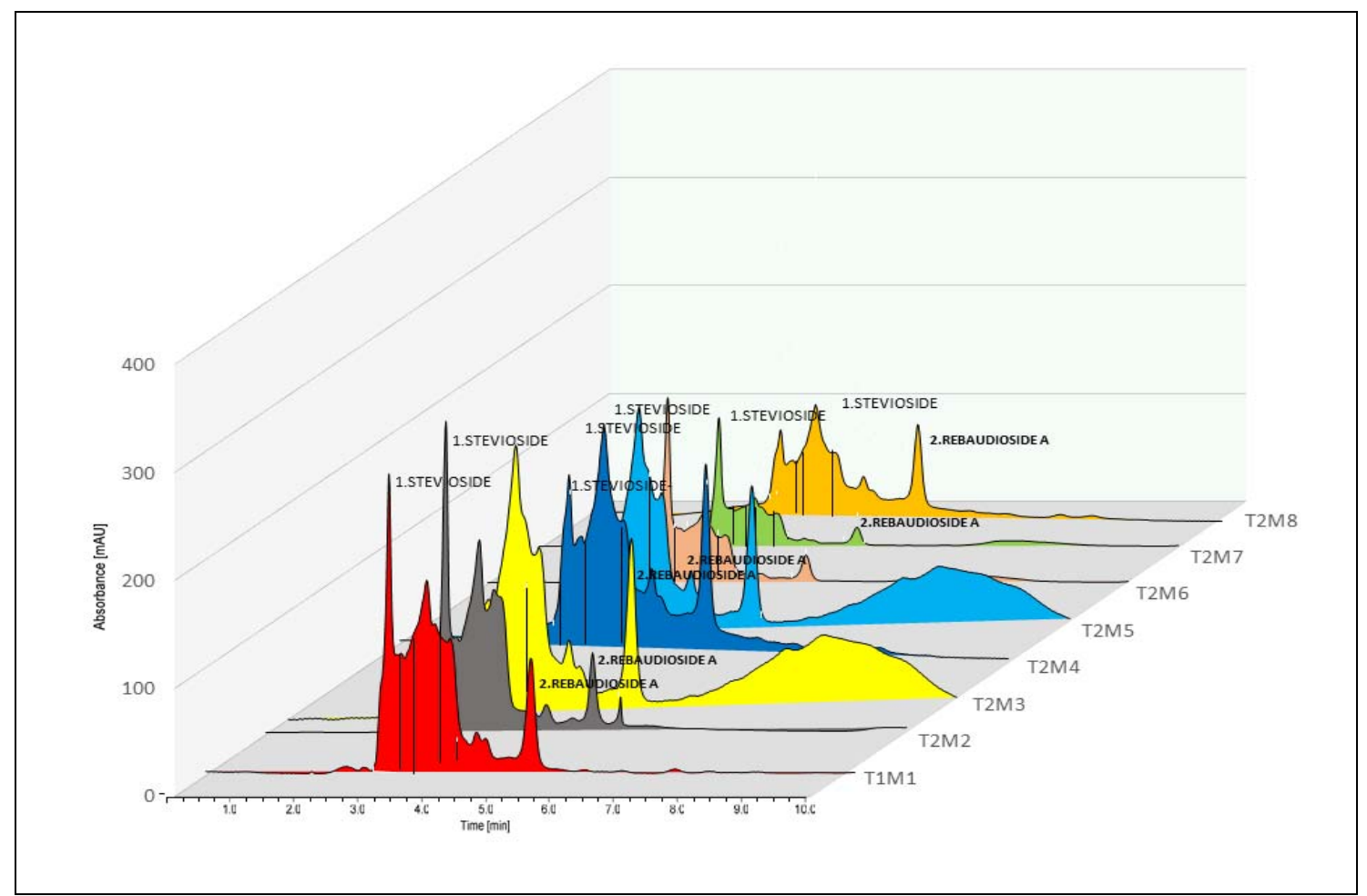

Fig. 2. LC-MS the effect of Biogas fertilizer and natural extracts method application on Stevioside and rebaudioside A., chromatogram of Stevia rebaudiana leaves in the first cut of the second season (2020). 


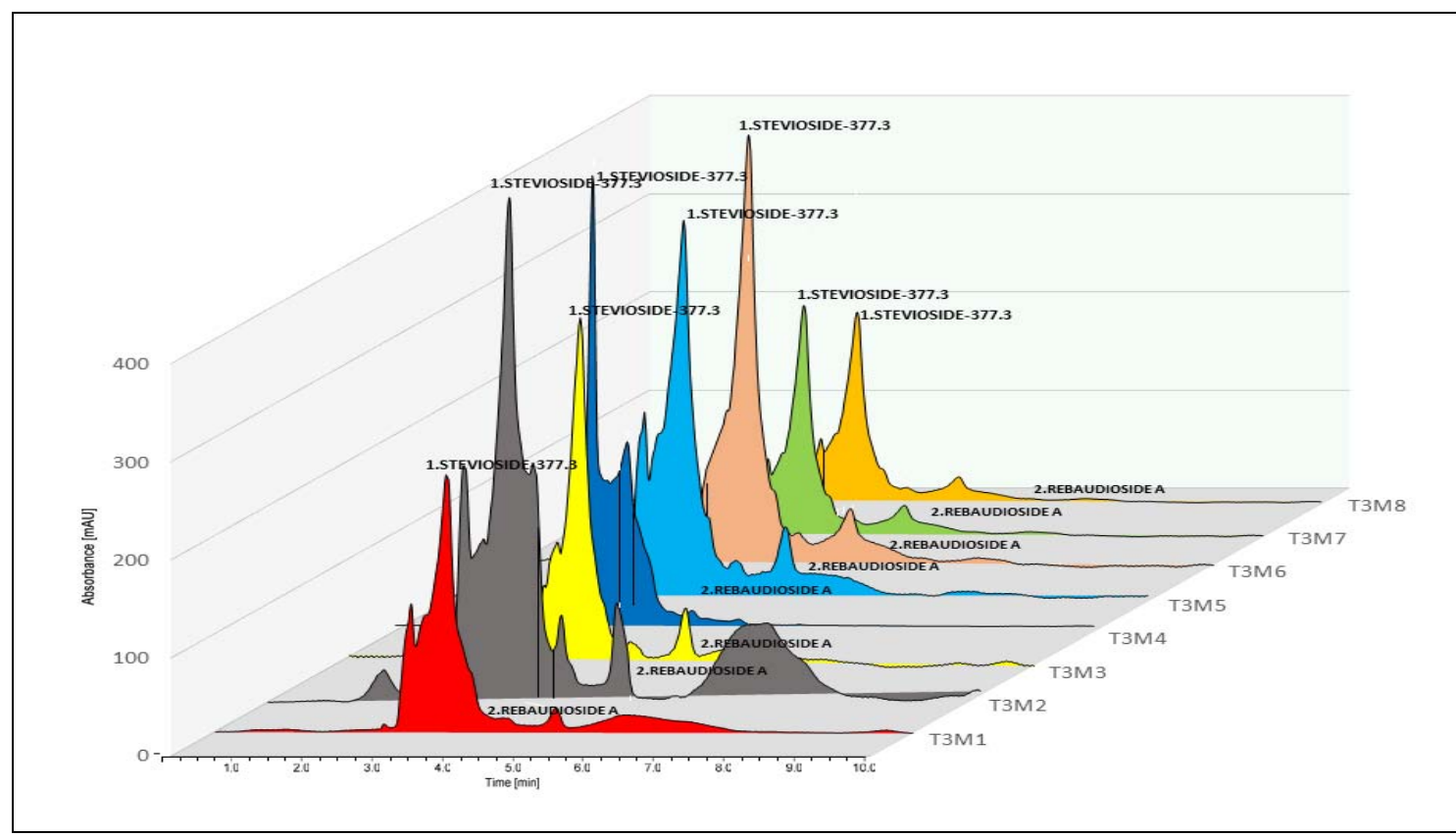

Fig. 3. LC-MS the effect of $50 \% \mathrm{NPK}+5000 \mathrm{~kg}$ biogas and natural extracts method application on Stevioside and rebaudioside A., chromatogram of Stevia rebaudiana leaves in the first cut of the second season (2020).

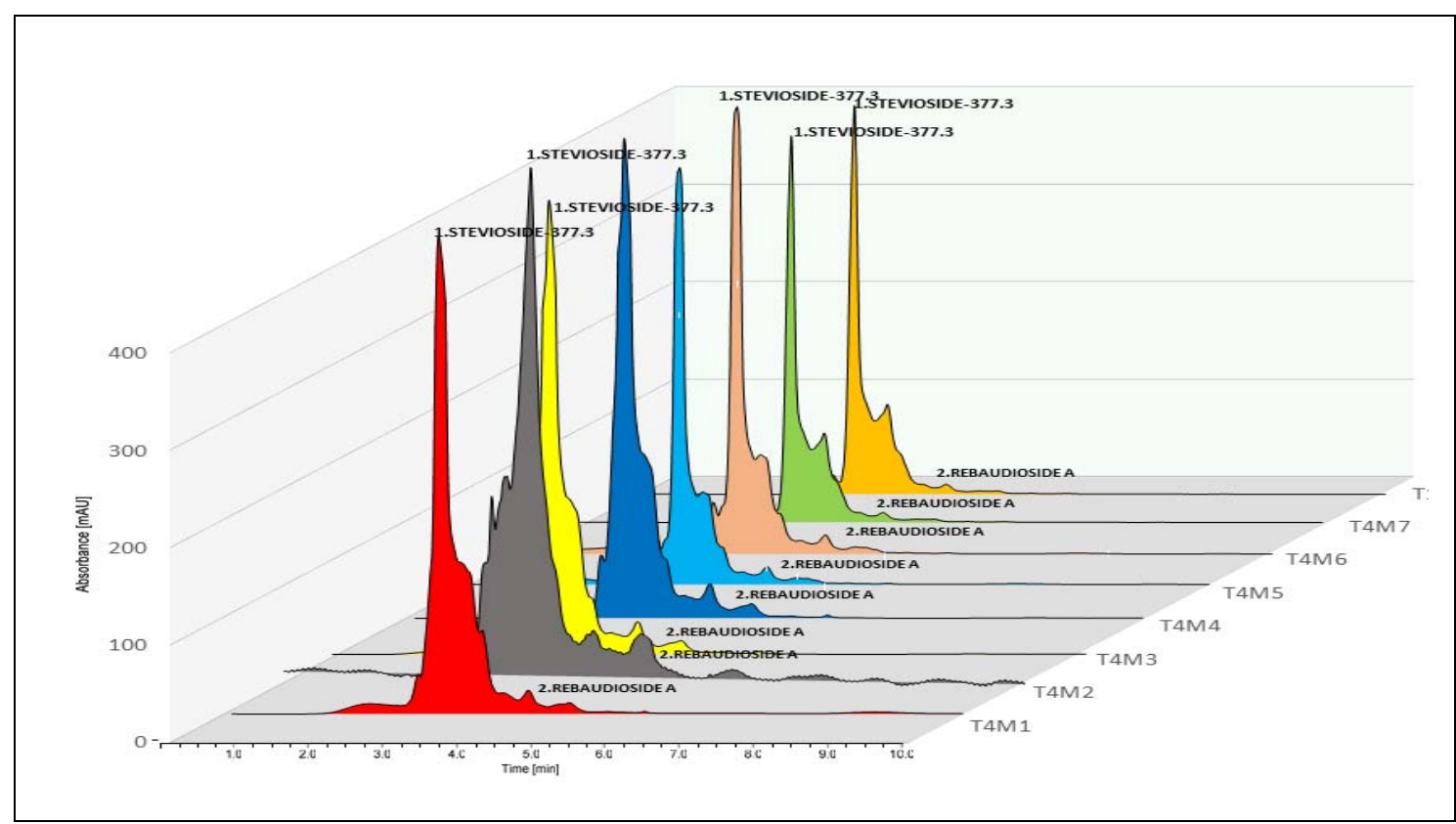

Fig. 4. LC-MS the effect of $50 \% \mathrm{NPK}+3000 \mathrm{~kg}$ biogas and natural extracts method application on Stevioside and rebaudioside A., chromatogram of Stevia rebaudiana leaves in the first cut of the second season (2020). 
In addition to improving soil quality, they supplied micro and macronutrients to the soil, increased nutrient uptake by plants, and increased plant growth. This was observed in biogas slurry application due to the supply of more readily available nutrients and the wider $\mathrm{C}: \mathrm{N}$ ratio of biogas slurry. That is why biogas slurry generally performs better in the later stages of its application in the soil in terms of nutrient availability, as it has a more consistent effect on nutrient uptake by plants due to its mineralization occurring later in the process and slowly release nutrients rather than providing nutrients to the plants in conjunction with mineral fertilizer This explains why better results are obtained in most cases with the second cut.

On the other hand combining, biogas slurry, a half dose of the chemical fertilizer with garlic extracts gave the highest values on the shoots fresh and dry yield in the second cut as a result of an increase in number of branches, number of leaves, the leaves surface area as this may be due to the biochemical function of vitamins and amino acids in the garlic aqueous extract that improves the role of metabolic processes and endogenous hormones (IAA and $\mathrm{GA}_{3}$ ) (Elzaawely et al., 2018). Extract of garlic resulted in cell propagation, cell expansion, and division of cells, contributing to an increase in the number of leaves. These effects can also be caused by the impact on the growth of the endogenous hormones all in treated plants of garlic extract; (Abd-Allah et al., 2021). These observations corresponded to (Ziedan and Eisa, 2016) on dill plant who concluded that using garlic extract with micronutrients to have better yield from fruit, it is easily available, environmentally safe and cost-effective, (Massoud et al., 2017) on Majorana hortensis L. (Massoud et al., 2019) on Caraway and (AbdelKader et al., 2014), on Salvia officinalis L.

Also the increases of photosynthetic pigment content, maybe due to a higher soil and nutrient retention and supply. This increase in photosynthetic pigment creation could also be a result of Azolla cytokinins' ability to prolong leaf aging by inhibiting chlorophyll breakdown and promoting protein and RNA synthesis (Castelfranco and Beale, 1983). These findings also corroborated with (Vanithamani, 2016) who stated that the greatest impact was exercised in organic fertilizer treatment (vermicompost) and half dose of NPK + Azolla. treatment revealed an increase in the photosynthetic pigments of chlorophyll (chlorophyll a, b and total chlorophylls) and carotenoids compared to untreated plants in Amaranthus polygonoids L.

Biogas slurry also contains organic nitrogen (primarily amino acids), abundant mineral elements, and low-molecular-mass bioactive substances (e.g. hormones, humic acids, vitamins, etc.) and may be due to bioactive compounds such as auxin, cytokinin, and gibberellins could be extracted from garlic and Azolla extracts as shown in this study which was used as an exogenous growth regulator. Whereas increased mineral content such as N.P.K may be due to the highest gibberellin $\left(\mathrm{GA}_{3}\right)$, auxin (IAA), and cytokinins (zeatin) contained in garlic and Azolla extract.

In this concern, the result is supported by the study of (Calvo et al., 2014), who stated that microbial inoculants could enhance nutrient uptake and thus improve the crop's nutrient status. As with fertilizers, biostimulants can help improve plant nutrition by stimulating natural mechanisms that aid in nutrient absorption and efficiency (De Pascale et al., 2017). (Al-karaawi, 2019) reported that administration of gibberellin $\left(\mathrm{GA}_{3}\right)$ may increase growth due to an increase in the effective leaf area hence photosynthesis increases. Cytokinins are compounds that can increase cell division in plant tissues and regulate plant growth and development, as well as kinetin, Additionally, soil-applied organic matter not only provides structure to the soil, but also provides energy for microbial activity that is necessary for nutrient recycling, affects 
nutrient availability such as $\mathrm{N}, \mathrm{P}, \mathrm{K}$, and $\mathrm{S}$, improves soil and water conservation, soil buffering and exchange capacity(Adebayo et al., 2017).

These organic fertilizers may accelerate plant growth in certain circumstances. The inclusion of growth promoters like auxin in biogas slurry is the primary rationale for this action (Warnars and Oppenoorth, 2014). Furthermore, it has been claimed that these can increase the permeability of the cell membrane, increasing water, and other nutritional element uptake. Additionally, water absorption and nitrogen content rose dramatically. Similarly, the inclusion of organic-based chemicals significantly increased root and shoot growth, as well as water uptake, in the N-free medium (Koszel and Lorencowicz, 2015).

On the other hand, the results obtained for stevia leaf nitrate concentrations indicated that while biogas slurry as an organic fertilizer decreased nitrate concentrations, the combination of biogas fertilizer and Azolla reduced nitrate concentrations to levels lower than those obtained with biogas alone in two seasons (Kawtar et al., 2017). It's worth noting that the nitrate levels observed in all treatments investigated are within regulatory limits. The former Scientific Committee on Food (SCF) established an average daily intake (ADI) for nitrate of $3.7 \mathrm{mg} / \mathrm{kg}$ body weight per day, equivalent to $222 \mathrm{mg}$ nitrate per day for a 60 $\mathrm{kg}$ adult, which was reconfirmed in 2002 by the Joint FAO/WHO Expert Committee on Food Additives (JECFA) and (E.F.S.A., 2008). Nitrate is benign at levels below the maximum residue levels (MRLs), but when it exceeds these levels, it can be harmful due to the decrease of nitrites, which can combine with amines and amides to form "N-nitroso" compounds that cause gastric cancer (SeyyedSalehi et al., 2021). These results agreed with (Rabie et al., 2020) on Matricaria Chamomilla who concluded that the addition of compost and 50\% of chemical fertilizer to Azolla extract at 50\% as foliar (spray Azolla) had a positive effect on chemical constituents except nitrate and nitrite concentrations. Meanwhile, (Fallah and Omrani, 2018) demonstrated that while nitrate concentrations in plants increased significantly following nitrogen fertilizer application, organic fertilizer use can lower nitrate concentrations in medicinal plants.

The glycosides accumulation of stevia leaves takes place throughout the life of the Stevia rebaudiana L., especially in the later stage of growth. At the latter growth stage, there is a large leaf area and a high net photosynthetic rate, which result in a significant buildup of photosynthesis product (Liu et al., 2011). Each physiological index is much higher in organic manure cultivation than in chemical fertilizer cultivation (Enchev et al., 2018). Rebaudioside A., has a weaker off-flavor than stevioside (Jung et al., 2021). Steviol glycosides with a superior taste are being investigated to generate sweeteners with a sensory profile that is closer to sucrose. The industry also uses rebaudioside A., which is now widely used (Rashwan and Ferweez, 2017). The ratio of rebaudioside A., to stevioside is the accepted measure of sweetness quality; the more rebaudioside $\mathrm{A}$., the better. If rebaudioside A., is present in equal quantities to stevioside, it appears that the after taste is eliminated (Majzoobi et al., 2018) To summarise, organic manure culture can enhance above-ground growth, root vigor, leaf net photosynthetic capacity, and total glycosides content in the leaf of Stevia. Thus, employing biogas fertilizer sparingly can improve the taste as reduces the sense of bitter taste. and quality of Stevia rebaudiana Bertoni, as well as improve the soil structure and safeguard the environment.

The results were in agreement with (Zaman et al., 2018) (Liu et al., 2011) (Rashwan and Ferweez, 2017) where the use of organic fertilizer improved the productivity and quality of stevia plants compared to chemical fertilizer, either by using organic fertilizer with half the dose of chemical fertilizer, which significantly affected the plant content of both stevioside 
and rebaudioside A., which led to obtaining the highest percentage of stevioside and the total glycosides. The increase in the total content of stevioside and rebaudioside may be due to the use of Azolla extract, which led to an increase in the leaf area, including enhancing the utilization of light energy and increasing the plant's chlorophyll content (Maswada et al., 2021) and enhancing the process of photosynthesis, which enhances the production of stevioside and rebaudioside.[(Sadegh Kasmaei et al., 2019); (Paler and Alcantara, 2021)].

\section{CONCLUSION}

Biogas used as organic fertilizer is characterized by an increase in nitrogen content, which improves the plant's chemical constituents, hence, increased yield and quality but it requires time for analysis, which were evident in the second cut of both seasons. The results indicated that substituting half of the recommended dose of chemical fertilizer with the same equivalent dose from the nitrogen content for biogas fertilizer resulted in a reduction of the problem of chemical fertilizer on human health included in nitrate accumulation and economic damages, in addition to the use of both Azolla and garlic extracts as a drench additive with organic fertilizer.

\section{REFERENCES}

A.O.A.C. (1980). Official Methods of Analysis, $13^{\text {th }}$ Ed. The Association of Official Analytical Chemists. Washington D.C., USA. 1018 p.

Abd El-hamid, N.S.M.; Makled, S.M.S. and Abd Elmonem, S.M.A. (2019). Determinants of production and export for some medicinal and aromatic plants in Egypt. Arab Universities Journal of Agricultural Sciences, 27(2):1351-1369.

Abd-Allah, W.; Khater, M.R. and El-Shafay R.M. (2021). Effect of spraying with extracts of plants and amino acids on growth and productivity on Coriandrum sativum L. plants under Shalateen condition. Plant Archives, 21:300-307.
AbdelKader, H.; Massoud, H.; Mosa, A.; Eisa, E. and Nour Eldeen, E. (2014). Effect of bio-fertilizers and plant extracts on growth, essential oil and chemical constituents of sage (Salvia officinalis L.) plant, under water stress conditions. Journal of Plant Production, 5(6):10031020 .

Abou Chehade, L.; Al Chami, Z.; De Pascali, S.A.; Cavoski, I. and Fanizzi, F.P. (2018). Biostimulants from food processing by-products: agronomic, quality and metabolic impacts on organic tomato (Solanum lycopersicum L.). Journal of the Science of Food and Agriculture, 98(4):1426-1436.

Abou Hussein, M.R.; Fadl, M.S. and Wally, Y.A. (1975 a). Effect of garlic bulb extract on flowering, sex ratio, and yield of squash, I. Effect of different fractions of partitioned garlic bulb extract on flowering in squash. Egyptian Journal of Horticulture, 2(1):3-10.

Abou Hussein, M.R.; Fadl, M.S. and Wally, Y.A. (1975 b). Effect of garlic bulb extract on flowering, sex ratio, and yield of squash, II. Modulation of sex ratio by application of different fractions of garlic bulb extract. Egyptian Journal of Horticulture, 2(1):11-22.

Abou-Arab, A.E.; Abou-arab, A.A. and AbuSalem, M.F. (2010). Physico-chemical assessment of natural sweeteners steviosides produced from Stevia rebaudiana bertoni plant. African Journal of Food Science 4(5):269-281.

Adebayo, A.G.; Akintoye, H.A.; Shokalu, A.O. and Olatunji, M.T. (2017). Soil chemical properties and growth response of Moringa oleifera to different sources and rates of organic and NPK fertilizers. International Journal of Recycling of Organic Waste in Agriculture, 6(4):281287.

Al Mayahi, M.Z. and Fayadh, M.H. (2015). The effects of garlic extract, its application methods and their interaction on growth and yield of potato, Solanum 
tuberosum (L.) cv. Latonia. Advances in Agriculture \& Botanics, 7(1):59-69.

Al-Karaawi, A.K.M. (2019). Effect of gibberellin $\mathrm{GA}_{3}$, boron and zinc foliar application on the growth and production of Hibiscus Sabdariffa L. Journal of Physics: Conference Series, Conference Series 1294(9):1-9. https://doi.org/10.1088/17426596/1294/9/092045

Andreuccetti, C.; Carvalho, R.A.; GaliciaGarcía, T.; Martínez-Bustos, F. and Grosso, C.R. (2011). Effect of surfactants on the functional properties of gelatinbased edible films. Journal of Food Engineering, 103(2):129-136.

Barrajón, C; Enrique, Herranz-López, M; Joven, J; Segura-Carretero, A; AlonsoVillaverde, C; Menéndez, J. A; and Micol, V. (2014). Molecular promiscuity of plant polyphenols in the management of age-related diseases: far beyond their antioxidant properties. Adv. Exp. Med. Biol., 824:141-59.

Benhmimou, A.; Ibriz, M.; Faïz, C.A.; Gaboun, F.; Douaik, A.; Amchra, F.Z.; Khiraoui, A. and Lage, M. (2017). Effects of planting density and harvesting time on productivity of natural sweetener plant (Stevia rebaudiana Bertoni.) in Larache Region, Morocco. International Journal of Plant Research, 7(4):83-89.

Bricker, B. (1991). MSTATC: A micro computer program from the design management and analysis of agronomic research experiments. Michigan State University.

Calvo, P.; Nelson, L. and Kloepper, J.W. (2014). Agricultural uses of plant biostimulants. Plant and Soil, 383(1):341.

Castelfranco, P.A. and Beale, S.I. (1983). Chlorophyll biosynthesis: recent advances and areas of current interest. Annual Review of Plant Physiology, 34(1):241-276.
Cataldo, D.A.; Haroon, M.H.; Schrader, L.E. and Youngs, V.L. (1975). Rapid colorimetric determination of nitrate in plant tissue by nitration of salicylic acid. Communications in Soil Science and Plant Analysis, 6(1):71-80.

Cottenie, A.; Verloo, M.; Kiekns, L.; Velghe, G. and Comer-lynek, R. (1982). Chemical analysis of plants and soil. Laboratory of Analytical and Agrochemistry, State University, Ghent, Belgium, $63 \mathrm{p}$.

De Pascale, S.; Rouphael, Y. and Colla, G. (2017). Plant biostimulants: Innovative tool for enhancing plant nutrition in organic farming. Eur. J. Hort. Sci., 82(6): 277-285.

Dekebo, A. (2019). Plant Extracts. IntechOpen, London, UK., 76 p.

Díaz-Gutiérrez, C.; Hurtado, A.; Ortíz, A.; Poschenrieder, C.; Arroyave, C. and Peláez, C. (2020). Increase in steviol glycosides production from Stevia rebaudiana Bertoni under organomineral fertilization. Industrial Crops and Products, 147:1-7. https://doi.org/10.1016/j.indcrop.2020.11 2220

E.F.S.A. (2008). Nitrate in vegetables-Scientific Opinion of the Panel on Contaminants in the Food chain. European Food Safety Authority Journal, 689:1-79.

El-Rokiek, K.G.; Dawood, M.G.; Sadak, M.S. and El-Awadi, M.E. (2019). The effect of the natural extracts of garlic or eucalyptus on the growth, yield and some chemical constituents in quinoa plants. Bulletin of the National Research Centre, 43 (1):119 -123.

El-Shimi, N.M.; El-Badawy, E.S.H.M. and Tolba, H.I. (2015). Response of sweet pepper plants to some organic and biofertilizers and its effect on fruit yield and quality. Middle East Journal of Agriculture Research, 4(3):435-445. 
Elzaawely, A.A.; Ahmed, M.E.; Maswada, H.F.; Al-Araby, A.A. and Xuan, T.D. (2018). Growth traits, physiological parameters and hormonal status of snap bean (Phaseolus vulgaris L.) sprayed with garlic cloves extract. Archives of Agronomy and Soil Science, 64(8):10681082.

Enchev, S.; Mehmed, A. and Kikindonov, G. (2018). Effect of mineral and organic fertilization on the productivity of stevia (Stevia rebaudiana b.). Bulgarian Journal of Agricultural Science, 24:98-100.

Enwall, K.; Philippot, L. and Hallin, S. (2005). Activity and composition of the denitrifying bacterial community respond differently to long-term fertilization. Applied and Environmental Microbiology, 71(12):8335-8343.

Fallah, S. and Omrani, B. (2018). Substitution of inorganic fertilizers with organic manure reduces nitrate accumulation and improves quality of purslane. Iranian Journal of Plant Physiology, 9(1):2651-2660.

Garg, R.N.; Pathak, H.; Das, D.K. and Tomar, R.K. (2005). Use of flyash and biogas slurry for improving wheat yield and physical properties of soil. Environmental Monitoring and Assessment, 107(1-3):1-9.

Hendawy, S.; Abouziena, H.; Abd El-Razik, T.; Amer, H. and Hussein, M. (2019). Winter weeds and its control in the medicinal plants in Egypt: a survey study. Egyptian Pharmaceutical Journal, 18(1):16-26.

Hossain, M.F.; Islam, M.T.; Islam, M.A. and Akhtar, S. (2017). Cultivation and uses of stevia (Stevia rebaudiana Bertoni): A review. African Journal of Food, Agriculture, Nutrition and Development, 17(4):12745-12757.

Jackson, M.L. (1959). Soil chemical analysis. Verlag: Prentice Hall, Inc., Englewood Cliffs, New Jersey, 85(3):251-252.
Jonas, H. (1969). Phytotoxicity of species of digitalis. Zeitschrift Fur Pflanzenphysiologie, 60(4): 359-369.

Jung, J.; Kim, S.; Park, S. and Hong, J.H. (2021). Sweetness profiles of glycosylated rebaudioside $\mathrm{A}$ and its binary mixtures with allulose and maltitol. Food Science and Biotechnology 2021 30(3):423-432.

Kawtar, A.E.; Ashour H.M. and Fatma, S.I. (2017). Growth characters and some chemical constituents of Matricaria chamomilla L. plants in relation to green manure and compost fertilizer in sandy soil. Middle East Journal of Agriculture Research, 6(1):76-86.

Khan, S.A.; Malav, L.C.; Kumar, S.; Malav, M.K. and Gupta, N. (2015). Resource utilization of biogas slurry for better yield and nutritional quality of baby corn. Advances in Environmental and Agricultural Science, 32:382-394.

Koszel, M. and Lorencowicz, E. (2015). Agricultural use of biogas digestate as a replacement fertilizers. Agriculture and Agricultural Science Procedia, 7:119124.

Liu, X.; Ren, G. and Shi, Y. (2011). The effect of organic manure and chemical fertilizer on growth and development of Stevia rebaudiana Bertoni. Energy Procedia, 5:1200-1204.

Lucini, L.; Rouphael, Y.; Cardarelli, M.; Bonini, P.; Baffi, C. and Colla, G. (2018). A vegetal biopolymer-based biostimulant promoted root growth in melon while triggering brassinosteroids and stress-related compounds. Frontiers in Plant Science, 9:1-11.

Majzoobi, M.; Mohammadi, M.; Mesbahi, G. and Farahnaky, A. (2018). Feasibility study of sucrose and fat replacement using inulin and rebaudioside $\mathrm{A}$ in cake formulations. Journal of Texture Studies, 49(5):468-475.

Massoud, G.F.; Dapor, A.S. and El-Shoura, A.M. (2019). Allelopathic effect of 
ecofriendly botanical extracts and application of vinasse as alternative source of mineral potassium fertilizers on yield and oil quality of Caraway plant. Middle East Journal of Agriculture Research, 8(4):1290-1305.

Massoud, H.; Sharaf-Eldin, M. and Sheashaa, A. (2017). Effect of biofertilizers, plants extracts and active dry yeast on marjoram plants. Journal of Plant Production, 8(5):657-663.

Maswada, H.F.; Abd El-Razek, U.A.; ElSheshtawy, A.N. and Mazrou, Y.S. (2021). Effect of azolla filiculoides on growth, physiological and yield attributes of maize grown under water and nitrogen deficiencies. Journal of Plant Growth Regulation, 40(2):558-573.

Mdlambuzi, T.; Muchaonyerwa, P.; Tsubo, M. and Moshia, M.E. (2021). Nitrogen fertiliser value of biogas slurry and cattle manure for maize (Zea mays L.) production. Heliyon, 7(5):1-6. https://doi.org/10.1016/j.heliyon.2021.e0 7077

Murphy, J. and Riley, J.P. (1962). A modified single solution method for the determination of phosphate in natural waters. Analytica Chimica Acta, 27:3136.

Naguib, N.Y.M. (2011). Organic vs chemical fertilization of medicinal plants: A concise review of researches. Advances in Environmental Biology, 5(2):394-400.

Paler, A.L.L.; and Alcantara, C.G. (2021). Effect of Azolla (Azolla pinnata) as a bio-stimulant on nutrient uptake and yield of mungbean (Vigna radiata L.). Organic Agriculture 11(3):469-475

Palhares, R.M.; Gonçalves Drummond, M.; dos Santos B.; Pereira Cosenza, G.; das Graças Lins Brandão, M. and Oliveira, G. (2015). Medicinal plants recommended by the world health organization: DNA barcode identification associated with chemical analyses guarantees their quality. Plos One,
10(5):1-29.

https://dx.doi.org/10.1371\%2Fjournal.po ne. 0127866

Piacente, S.; Pizza, C. and Oleszek, W. (2005). Saponins and phenolics of yucca schidigera roezl: chemistry and bioactivity. Phytochemistry Reviews, 4(2-3):177-190.

Rabie, K.A.; El-Sherif, M.H.; El-Shahat, R.M. and Ali, F.S. (2020). Azolla as an alternative of mineral nitrogen for chamomile plant (Matricaria chamomilla L.) fertilization in sandy soil. Asian Journal of Soil Science and Plant Nutrition, 5(4):1-13.

Rashwan, B. and Ferweez, H. (2017). Effect of mineral nitrogen fertilization and compost on stevia yield and its profitability. Journal of Soil Sciences and Agricultural Engineering, 8(6):215-222.

Rayment, G.E. and Lyons, D.J. (2010). Soil Chemical Methods-Australasia. Csiro Publishing, Australia, 520 p.

Roba, T.B. (2018). The effect of mixing organic and inorganic fertilizer on productivity and soil fertility. Open Access Library Journal, 5(6):1-11.

Sadegh Kasmaei, L.; Yasrebi, J.; Zarei, M.; Ronaghi, A.; Ghasemi, R.; Saharkhiz, M. J; Ahmadabadi, Z. and Schnug, E. (2019). Influence of plant growth promoting rhizobacteria, compost, and biochar of azolla on rosemary (Rosmarinus officinalis L.) growth and some soil quality indicators in a calcareous soil. Communications in Soil Science and Plant Analysis, 50(2):119131.

Saric, M. (1967). Chlorophyll Determination. Univerzitet $U$ Noveon Sadu Praktikum Iz Fiziologize BiljakaBeograd. Hauena Anjiga, 215 p.

Seyyed Salehi, M.S.; Mohebbi, E.; Sasanfar, B.; Toorang, F. and Zendehdel, K. (2021). Dietary N-nitroso compounds intake and bladder cancer risk: $\mathrm{A}$ systematic review and meta-analysis. 
Nitric Oxide - Biology and Chemistry, 115:1-7.

Singh, D.P.; Kumari, M.; Prakash, H.G.; Rao, G.P. and Solomon, S. (2019). Phytochemical and pharmacological importance of stevia: A calorie-free natural sweetener. Sugar Tech, 21(2):227-234.

Snedecor, G.W. and Cochran, W.G. (1989). Statistical Methods, $6^{\text {th }}$ Ed. Ames., Iowa, Iowa State University Press Section, 12:349-352.

Sonklien, C.; Intanon, P.; Terapongtanakorn, S. and Intanon, R. (2020). Comparative assessment of fertilizers on yield and quality of marigold (Tagetes erecta L.). Indian Journal of Agricultural Research, 54(3):367-372.

Synge, R.L.M. (1970). Proteins and poisons in plants. Chemiker-Zeitung, 94(17):647.

Talevi, A. (2021). Potential medicinal effects and applications of stevia constituents. Phytochemistry Reviews. https://doi.org/10.1007/s11101-02109753-5

Vanithamani, J. (2016). Impact of individual and combined exposure of bio-fertilizer, chemical fertilizer and vermicompost on the biochemical constituents of Amaranthus polygonoids L. J. Vanithamani, 3(2):170-180.

Warnars, L. and Oppenoorth, H. (2014). Bioslurry: A Supreme Fertiliser, A Study on Bioslurry Results and Uses. Hivos People Unlimited, $48 \mathrm{p}$.

Willer, H. and Lernoud, J. (2019). The world of organic agriculture. Statistics and emerging trends. Research Institute of Organic Agriculture FiBL and IFOAM Organics International $336 \mathrm{p}$.

Wilson, G. and Al-Hamdani, S. (1997). Effects of chromium (vi) and humic substances on selected physiological responses of Azolla caroliniana. American Fern Journal, 87(1):17-27.

Yamika, W.S.; Herlina, N. and Amriyanti, S. (2019). The effect of biogas slurry and inorganic fertilizer on soil fertility and yield of cucumber (Cucumis sativus L.). Journal of Degraded and Mining Lands Management, 6(4):1829-1835.

Yan, L.; Liu, C.; Zhang, Y.; Liu, S. and Zhang, Y. (2021). Effects of $\mathrm{C} / \mathrm{N}$ ratio variation in swine biogas slurry on soil dissolved organic matter: Content and fluorescence characteristics. Ecotoxicology and Environmental Safety, 209:1-12. https://doi.org/10.1016/j.ecoenv.2020.11 1804

Zaman, M.; Rahman, M.A.; Chowdhury, T. and Chowdhury, M. (2018). Effects of combined application of chemical fertilizer and vermicompost on soil fertility, leaf yield and stevioside content of stevia. Journal of the Bangladesh Agricultural University, 16(1):73-81.

Ziedan, E. and Eisa, E. (2016). The use of some micronutrients and plant extracts of resistance to powdery mildew and nutrition dill plants in the gharbiyah governorate. Journal of Plant Protection and Pathology, 7(9):579-586.

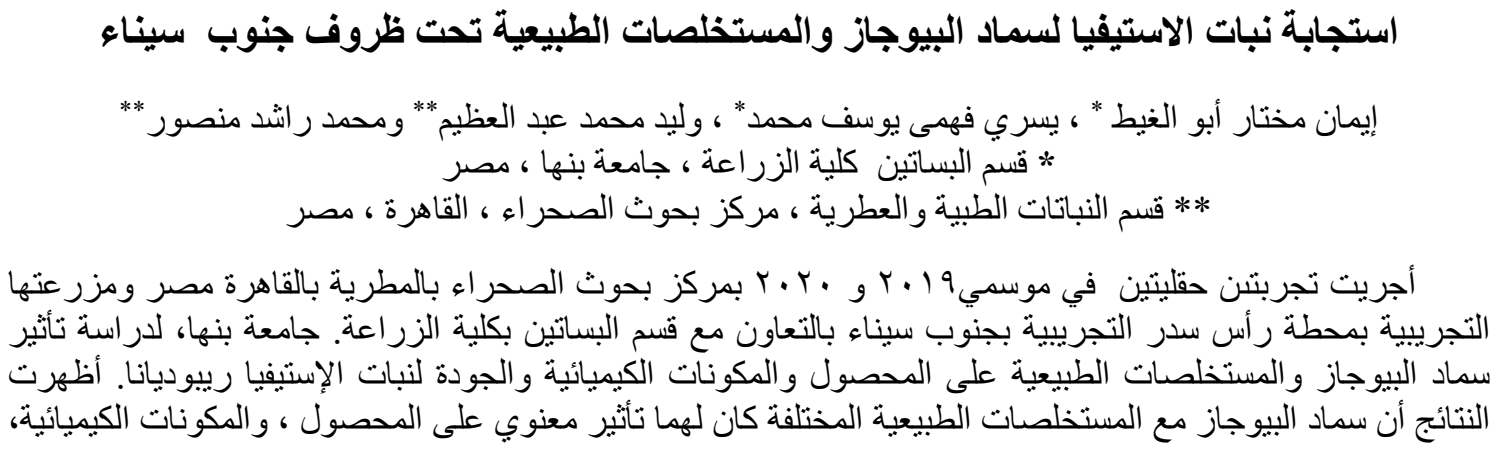




\section{Eman M. Abou El-Ghait et al.}

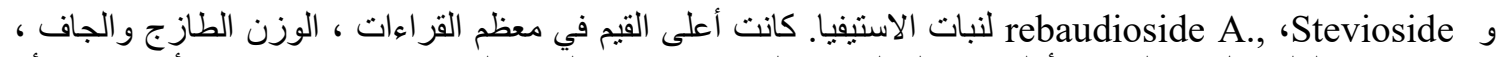

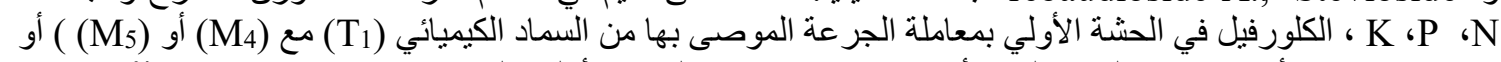

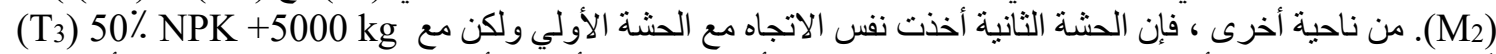
لمت ha-1

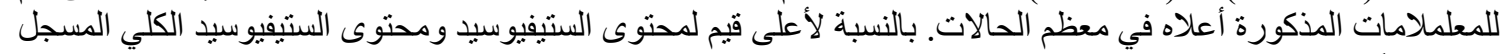

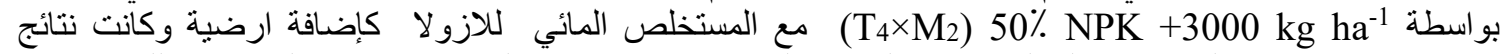

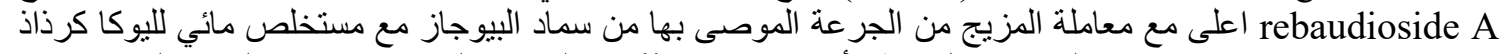

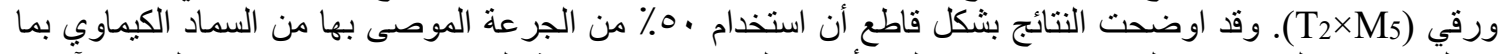

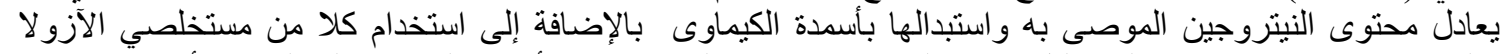

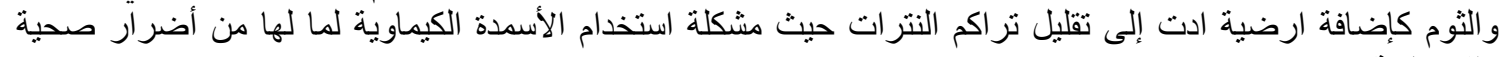
و اقتصادية. 